\title{
1 Title: Perceptual gating of a brainstem reflex facilitates speech 2 understanding in human listeners
}

Heivet Hernandez-Perez * ${ }^{1}$, Jason Mikiel-Hunter ${ }^{1}$, David McAlpine ${ }^{1}$, Sumitrajit Dhar ${ }^{2}$, Sriram

5 Boothalingam ${ }^{3}$, Jessica J.M. Monaghan $4 \$$, Catherine M. McMahon ${ }^{1 \ddagger}$

61 The Australian Hearing Hub, Macquarie University, Australia ${ }^{2}$ Department of Communication

7 Sciences and Disorders, Northwestern University, USA ${ }^{3}$ University of Wisconsin-Madison, USA ${ }^{4}$

8 National Acoustic Laboratories, Australia

$9 \quad \ddagger$ C.M.M and J.J.M.M contributed equally to this work

10 * Corresponding author

11 Heivet Hernandez-Perez.

12 Email: heivet.hernandez-perez@mq.edu.au

\section{Abstract}

15 Navigating "cocktail party" situations by enhancing foreground sounds over irrelevant background

16 information is typically considered from a cortico-centric perspective. However, subcortical circuits,

17 such as the medial olivocochlear (MOC) reflex that modulates inner ear activity itself, have ample

18 opportunity to extract salient features from the auditory scene prior to any cortical processing. To

19 understand the contribution of auditory subcortical nuclei and the cochlea, physiological recordings

20 were made along the auditory pathway while listeners differentiated non(sense)-words and words.

21 Both naturally-spoken and intrinsically-noisy, vocoded speech - filtering that mimics processing

22 by a cochlear implant-significantly activated the MOC reflex, whereas listening to speech-in-

23 background noise revealed instead engagement of midbrain and cortical resources. An auditory

24 periphery model reproduced these speech degradation-specific effects, providing a rationale for

25 goal-directed gating of the MOC reflex to enhance representation of speech features in the auditory

26 nerve. Our data reveals the co-existence of two strategies in the auditory system that may facilitate

27 speech understanding in situations where the speech signal is either intrinsically degraded or

28 masked by extrinsic auditory information. 


\section{Introduction}

31 Cocktail-party listening, the ability to focus on a single talker in a background of simultaneous,

32 overlapping conversations, is critical to human communication, and a long-sought goal of hearing

33 technologies [1,2]. Problems listening in background noise are a key complaint of many listeners

34 with even mild hearing loss, and a stated factor in the non-use and non-uptake of hearing devices

35 [3-5]. However, despite its importance in every-day listening tasks, and its relevance to hearing

36 impairment, physiological mechanisms that enhance attended speech remain poorly understood.

37 In addition to local circuits in the auditory periphery and brainstem that have evolved to enhance

38 automatically the neural representation of ecologically relevant sounds [6-8], it is likely that such a

39 critical goal-directed behaviour as cocktail-party listening also relies on top-down, cortically-driven

40 processes to emphasize perceptually relevant sounds, and suppress those that are irrelevant

$41[9,10]$. Nevertheless, the specific role of bottom up and top-down mechanisms in complex listening

42 tasks remain to be determined.

43 A potential mechanistic pathway supporting cocktail party listening is the auditory efferent system,

44 whose multi-synaptic connections extend from auditory cortex (AC) to the inner ear [11-13]. In

45 particular, reflexive activation of fibers in the medial olivocochlear (MOC) reflex innervating the

46 outer hair cells (OHCs)-electromotile elements responsible for the cochlea's active amplifier-is

47 known to reduce cochlear gain [14], thereby increasing the overall dynamic range of the inner ear

48 and facilitating sound encoding in high levels of background noise [15].

49 MOC fibers (ipsilateral and contralateral to each ear) originate in medial divisions of the superior

50 olivary complex in the brainstem and synapse directly on the basal aspects of OHCs (Warr and

51 hand neural sensitivity to sound $[16,17]$.

$52 \mathrm{MOC}$ neurons are also innervated by descending fibers from $\mathrm{AC}$ and midbrain neurons, providing

53 potential means of modulating cochlear activity in a task-related or attention-dependent manner

54 [18-20]. Although it has been speculated that MOC-mediated changes in cochlear gain might

55 enhance speech coding in background noise [21], the role of the MOC reflex in reducing cochlear

56 gain during goal-directed listening in normal hearing human subjects (i.e., with physiologically 
57 normal $\mathrm{OHCs}$ ) remains controversial. In particular, it remains unclear under which conditions MOC

58 reflex is active, including whether listeners must actively be engaged in listening task [22-24].

59 Efferent-mediated changes in cochlear gain can be assessed by measuring otoacoustic emissions

60 (OAEs), energy generated by the active OHCs and measured non-invasively as sound from the

61 ear canal [25]. When transient sounds, such as clicks, are delivered to one ear in the presence of

62 noise in the opposite ear; OAE amplitudes are reduced, reflecting increased MOC efferent activity

63 [26]. The magnitude of OAE s has been reported as either increased [23,27], reduced [22,28] or

64 unaffected $[29,30]$ in participants with improved speech-in-noise perception. Confounding effects

65 on cochlear gain could depend on factors such as task difficulty or relevance (e.g., speech vs. non-

66 speech tasks) or even methodological differences in recording and analysing inner ear signatures

67 such as OAEs [23,24].

68 Here, we examined the role of the auditory efferent system in active (participant's attention directed

69 towards the speech stimuli) vs. passive (participant's attention directed away from the speech

70 stimuli and towards a silent, non-subtitled film) listening conditions for three commonly employed

71 speech manipulations: vocoding of 'natural' speech— filtering that mimics processing by a cochlear

72 implant; speech presented in a background of multi-talker 'babble' noise, and speech presented in

73 a background of speech-shaped noise i.e. noise with the same long-term spectrum as speech.

74 Physiological recordings in the central auditory pathway, including brainstem, midbrain and cortical

75 responses were made whilst listeners performed an active listening task (detecting non-words in a

76 string of Australian-English words and non-words). Our experimental paradigm was designed to

77 maintain fixed levels of task difficulty that allowed us to preserve comparable task relevance across

78 the speech manipulations and therefore avoid confounding effects of task difficulty on top-down

79 modulation of activation of the MOC reflex. In addition, homologous visual and auditory scenes

80 were implemented to control for differences in alertness between active and passive listening

81 conditions.

82 When task difficulty was maintained across speech manipulations, measures of hearing function at

83 the level of the cochlea, brainstem, midbrain and cortex were modulated differentially depending

84 on the type of degradation applied to speech sounds, and on whether or not speech was actively 
85 attended. Specifically, the MOC reflex, assessed in terms of the magnitude of click-evoked OAEs

86 (CEOAEs), was activated by vocoded speech—an intrinsically degraded speech signal—but not

87 by otherwise 'natural' speech presented in either babble-noise or speech-shaped noise.

88 Furthermore, neural activity generated by the auditory midbrain was significantly increased in active

89 vs. passive listening for speech in babble and speech-shaped noise, but not for vocoded speech.

90 This increase was associated with elevated cortical markers of listening effort for the speech-in-

91 noise conditions. A model of the auditory periphery including an MOC circuit with biophysically

92 realistic temporal dynamics confirmed the stimulus-dependent role of the MOC reflex in enhancing

93 neural coding of speech signals. Our data suggest that otherwise identical performance in active

94 listening tasks may invoke quite different efferent circuits, requiring different levels of listening effort,

95 depending on the type of stimulus degradation experienced.

96 


\section{Results}

\section{Maintaining task relevance across speech manipulations requires iso-performance}

99 We assessed speech intelligibility—specifically the ability to discriminate between Australian-

100 English words and non-words-when speech was degraded by three different manipulations:

101 noise-vocoding the entire speech waveform; adding 8-talker babble noise to 'natural' speech (i.e.

102 speech-in-quiet) and adding speech-shaped noise to 'natural' speech. Participants were asked to

103 respond (by means of a button press) when they heard a non-word in a string of words and non-

104 words (Figure1A).

105 Three levels of task difficulty were achieved by altering either the number of noise-vocoded 106 channels-16 (Voc16), 12 (Voc12) and 8 (Voc8) channels)-or by altering the signal-to-noise ratio 107 (SNR) when speech was masked by babble noise-+10 (BN10) and +5 (BN5) dB SNR-or speech108 shaped noise-+8 (SSN8) and +3 (SSN3) dB SNR (Figure 1B). This was statistically confirmed in 109 all 56 listeners ( $n=27$ in the vocoded condition, and $n=29$ in the two masked conditions) who

110 showed consistently better performance-a higher rate of detecting non-words-in the less

111 degraded conditions i.e. more vocoded channels or higher SNRs in the masked manipulations

112 (Repeated Measures ANOVA (rANOVA) [vocoded: $[F(3,78)=70.92, p=0.0001]$; babble noise:

$113[F(3,78)=70.92, p=0.0001]$ and speech-shaped noise: $[F(2,56)=86.23, p=0.0001]$, post hoc

114 analysis with bonferroni corrections (6 multiple comparisons for the noise-vocoded experiment and 1153 for BN and SSN manipulations), Figure 1B.

116 Iso-performance was achieved across speech manipulations, with best performance observed in

117 the two natural speech conditions-one employed in the vocoding experiment and the other in the

118 two masked conditions: (One-way ANOVA $[F(1,54)=0.43, p=0.84]$ ). A moderate and similar

119 level of performance (significantly lower than performance for natural speech) was achieved across

120 Voc16/ Voc12 (n.s. compared to each other; $p=0.11$ ), BN10 and SSN8 conditions: (One-way

121 ANOVA: $[F(3,108)=0.67, p=0.57])$. The poorest performance, significantly lower than the high 122 and moderate performance levels, was observed for Voc8, BN5 and SSN3: $([F(3,81)=0.07, p=$ $1230.72])$. 
A

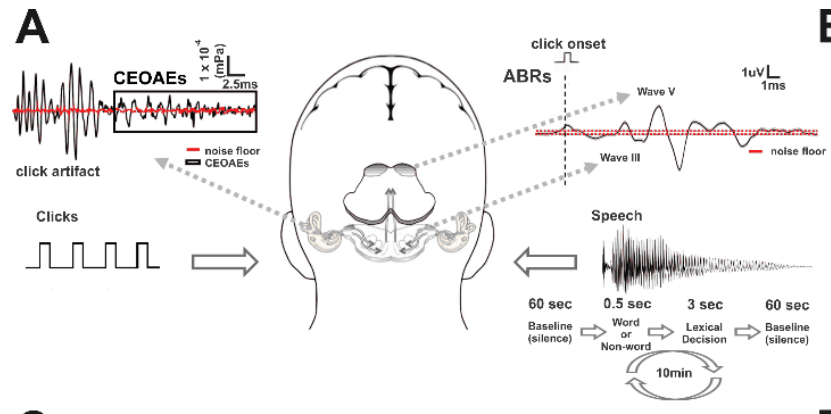

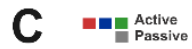

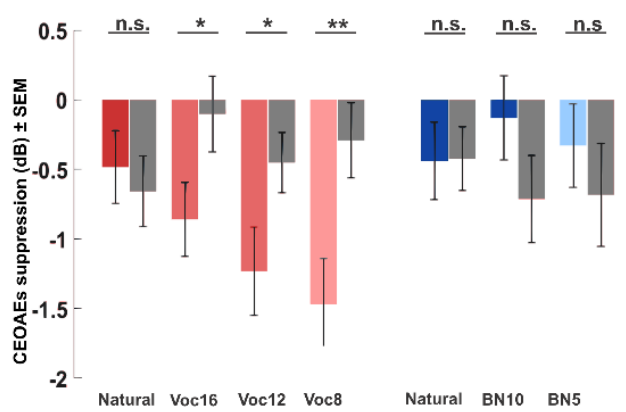

B

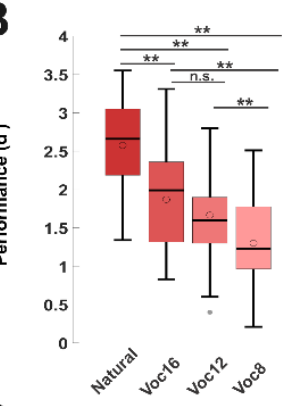

D Noise Vocoded

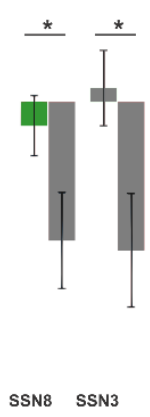

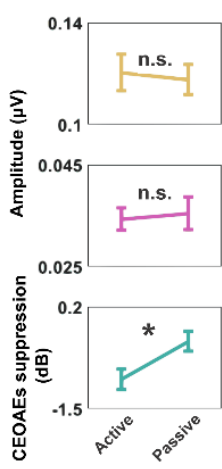

Babble Nois
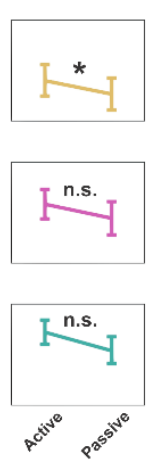
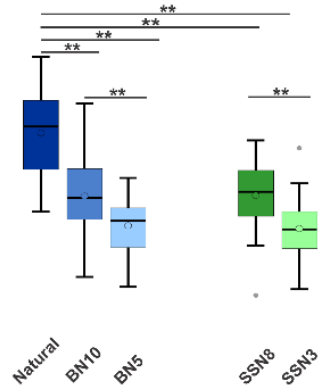

SpeechShaped Noise
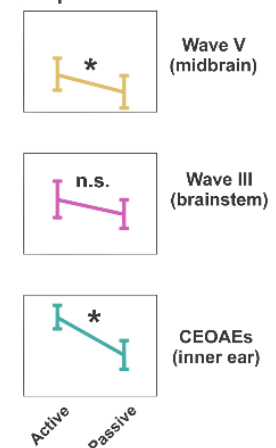

Figure 1. Behavioural and physiological measurements during active and passive listening. A. Schematic of the experimental paradigm (adapted with permissions, [31]). Clicks were continuously presented in one ear whereas speech tokens were delivered contralaterally in the other ear for 10 minutes to evoke CEOAEs. One minute of baseline CEOAEs (only clicks being delivered) were always recorded at the beginning and at the end of each condition. Participants had $3 \mathrm{sec}$ to make a lexical-decision (discriminate words vs. non-words) in the active listening condition whereas in the passive condition they were asked to ignore all the auditory stimuli and watch a movie. Upper left panel shows the CEOAEs analysis window obtained from the inner ear recordings. Upper right panel corresponds to click-evoked ABRs recordings. B. Performance during the lexical-decision task. Mean d' is represented in white circles ( $n=27$ in the noise-vocoded condition, and $n=29$ in the two masked conditions), median corresponds to the horizontal line. The upper and lower limits of the boxplot represent the $1^{\text {st }}(q 1)$ and $3^{\text {rd }}$ (q3) quartiles respectively while the whiskers denote the interquartile range (IQR $=q 3-q 1)$. Within speech conditions comparisons showed that the highest performance was always achieved for natural speech compared to the all noise-vocoded, babble noise and speech-shaped noise manipulations (bonferroni corrected $\mathrm{p}=0.001$ ). Performance was moderately-high (statistically lower than natural speech but higher than Voc8, BN5 and SSN3) for Voc16 and Voc12 (non-significant differences (n.s.), bonferroni corrected $p=0.11$ ) as well as for BN10 and SSN8 respectively. The lowest level of performance was predictably observed for Voc8, BN5 and SSN3 $(p=0.001)$. C. Click-Evoked OAEs suppression. The figure shows mean CEOAE magnitude changes relative to the baseline for all conditions. D. CEOAEs and ABRs collapsed (rANOVA main effect of Conditions (Active and Passive)). CEOAEs and ABRs are reported as means \pm SEM. $\left({ }^{* *}\right.$ bonferroni corrected $p<0.01$; ${ }^{*}$ bonferroni corrected $\left.p<0.05\right)$. 
145 As increasing task difficulty has been previously linked to the allocation of auditory attention and

146 cognitive resources towards the task itself [32], we employed the discrete and matching levels of

147 task difficulty across speech manipulations as a proxy for the required auditory attention.

\section{MOC reflex is modulated by task engagement in a stimulus-dependent manner}

149 To determine whether auditory attention modulates cochlear gain via the auditory efferent system

150 in a task-dependent manner, we assessed the effect of active vs. passive listening and speech

151 manipulation on inner ear activity. Click-evoked OAEs (CEOAEs) were recorded continuously

152 whilst participants both actively performed the lexical task, and passively listened to the same

153 corpus of speech tokens (Figure 1A). Compared to baseline measures obtained in the absence of

154 speech (in the ipsilateral ear but click stimuli in the contralateral one), CEOAEs were significantly

155 reduced in magnitude when actively listening to natural speech, and in all noise-vocoded conditions

156 (natural: [t $(24)=-2.33, p=0.03]$; Voc16: [t $(23)=3.40, p=0.002]$; Voc12: [t $(24)=3.98, p=0.001]$

157 and Voc8: [t $(25)=5.14, p=0.001])$. Conversely, during passive listening, CEOAEs obtained during

158 natural, but not noise-vocoded, speech were significantly smaller than baseline: [t $(25)=2.29, p=$

159 0.03], as were CEOAEs recorded during the two masked conditions at all SNRs (natural: [t $(26)=$

$1602.17, p=0.04]$; BN10: [t $(28)=2.80, p=0.009$ ] and BN5: [t $(28)=2.36, p=0.02]$; SSN8: [t $(28)=$

$1613.37, p=0.002]$ and SS3: [t $(28)=3.50, p=0.002])$. This suggests that auditory efferent activity is

162 modulated differently in active and passive listening, and by the different speech manipulation

163 types, despite iso-performance across experiments.

164 We calculated the reduction in CEOAEs between baseline and experimental conditions (CEOAE 165 suppression) - a proxy for activation of the MOC reflex-to quantify auditory efferent control of 166 cochlear gain in active and passive listening. For noise-vocoded speech, suppression of CEOAEs 167 was significantly greater when participants were actively engaged in the lexical task $(-1.01 \pm 0.18$ $168 \mathrm{~dB})$ compared to when they were asked to ignore the auditory stimuli $(-0.38 \pm 0.18 \mathrm{~dB})$ : rANOVA:

$169[\mathrm{~F}(1,22)=8.49, \mathrm{p}=0.008]$, (Figure 2A). Moreover, a significant interaction was observed between 170 conditions and stimulus-type: $[F(3,66)=2.80, p=0.046]$, indicating that the suppression of 171 CEOAEs was stronger for all vocoded conditions in which listeners where required to make lexical 172 decisions, compared to when they were not-Voc16: ([t $(23)=-2.16, p=0.04]$; Voc12: [t $(24)=-$ 
173 2.19, $p=0.038]$ and Voc8: [t $(25)=3.51, p=0.002])$. Task engagement did not alter CEOAE

174 suppression for natural speech [t $(24)=0.62, p=0.54]$.

175 In contrast, speech in speech-shaped-noise elicited the opposite pattern of results to noise-

176 vocoded speech (Figure 1C). The suppression of CEOAEs was significantly stronger during

177 passive $(-0.83 \pm 0.26)$ compared to active listening $(-0.16 \pm 0.21)$ : $r A N O V A$ : $[F(1,24)=4.44, p=$

178 0.046]. A significant interaction between condition and stimulus-type: $[F(2,48)=4.67, p=0.014]$

179 confirmed this for both SNRs: (SSN8 [t $(27)=2.71, p=0.01]$ and SSN3 [t $(28)=2.67, p=0.012]$ )

180 (Figure 1C). Intermediate effects on CEOAEs were observed for words masked by babble noise,

181 with CEOAE significantly smaller than baseline measures during passive, but not active, listening.

182 Cochlear gain was therefore suppressed during active listening of noise-vocoded speech, slightly

183 but significantly suppressed during passive listening in babble noise, and strongly suppressed

184 during passive listening in speech-shaped-noise. Together, our data suggest that the MOC reflex

185 is modulated by task engagement, and strongly depends on the way in which signals are degraded

186 including the type of noise used to mask the speech.

187 Auditory brainstem activity reflects changes in cochlear gain when listening to speech-in188 noise

189 The effects of active vs. passive listening on the cochlear gain were evident in the activity of 190 subcortical auditory neurons when we simultaneously measured auditory brainstem responses 191 (ABRs) to the same clicks used to evoke CEOAEs.

192 Click-evoked ABRs_-measured during presentation of speech-in-noise—showed similar effects to 193 those observed for CEOAE measurements. Specifically, in both masked conditions wave V194 corresponding to neural activity generated in the midbrain by the inferior colliculus (IC) and was 195 significantly enhanced in the active compared to the passive listening condition (Figure 1C) (speech 196 in babble noise: $[F(1,26)=5.67, p=0.025]$ and speech-shaped noise: $[F(1,26)=9.22, p=$ 197 0.005]). No changes in brainstem or midbrain activity were observed between active and passive 198 listening of noise-vocoded speech.

199 To rule out that the differing results were due to intrinsic differences in the two populations tested 200 (noise-vocoded Vs. masked speech experiments), we compared CEOAE suppression and ABR 
201 wave amplitudes in the two groups for active and passive listening of natural speech . No statistical 202 differences were observed for either active or passive listening, therefore, the differences observed 203 in both cochlear gain and auditory brainstem Imidbrain activity here can be attributed to the speech 204 degradation presented (active: suppression of CEOAEs [t (23) $=-0.21, p=0.83$; wave III [t (23) = $2050.45, p=0.65]$; wave $\vee[t(23)=0.09, p=0.93$ ]; passive: suppression of CEOAEs [t $(24)=-0.36$,

$206 \mathrm{p}=0.72$ ]; wave III [t (24) $=-0.16, \mathrm{p}=0.88$ ]; wave V [t (26) $=0.40, \mathrm{p}=0.69]$ ).

207 Together with our CEOAEs results, our data suggests that reduced ABR magnitudes appear only 208 inherited from reduction of the cochlear gain for masked stimuli. These results in the midbrain 209 indicate that while local cochlear gain changes are sufficient when processing single degraded 210 streams such as noise-vocoded speech, processing masked speech requires the involvement of 211 higher-level auditory structures.

212 Simulated MOC reflex improves the neural representation of vocoded speech, but not 213 speech-in-noise

214 To understand why the control of cochlear gain appears to depend on how speech is degraded, 215 we implemented a model of the auditory periphery incorporating MOC reflex through a brainstem 216 circuit with biophysically-realistic temporal dynamics [33]. We specifically tested the hypothesis that 217 suppression of cochlear gain enhances neural encoding of the stimulus envelope via the activation 218 of the MOC reflex. To assess how cochlear gain suppression affects neural representation of 219 speech in AN fibers, natural and degraded (those generating iso-performance in the active task 220 (Voc8; BN5; SSN3)) speech tokens were presented individually to the model at $75 \mathrm{dBA}$, with and 221 without MOC reflex (Figure 2A). We performed this analysis first for AN fibers with low-spontaneous 222 rate, and high-thresholds given their crucial role in detecting signals in noise as well as their lack 223 of saturation at high intensity levels [34-36].

224 The neural cross-correlation coefficient, $\rho E N V$, a measure of how similar neural envelopes are in 225 different AN spike train responses [37], was employed to quantify the effect of introducing the MOC 226 reflex on envelope encoding at seven logarithmically-spaced frequencies between $1-2 \mathrm{kHz}$. Values

227 of $\rho E N V$, ranging from 0 to 1 for independent to identical neural envelopes respectively, were 228 calculated for the three speech manipulations before and after the MOC reflex was included, with 
229 the neural envelope for natural speech acting as the "control" template for comparison. Natural 230 speech "control" simulations were always performed with the MOC reflex, given our observations

231 that a steady CEOAE suppression-indicative of an active MOC reflex- occurred for natural

232 speech experimentally (Figure 1C) and that neural envelopes for natural stimuli were enhanced in 233 model AN fibres with an MOC reflex present (Figure supplement 2).

234 For the example word, 'Fuzz', the three speech degradations in the absence of MOC reflex showed

235 high average similarity in their neural envelopes to their natural speech controls (Figure 2A) (mean

$236 \rho E N V_{A N}$ for $\operatorname{Voc} 8=0.84 \pm 0.01 ;$ mean $\rho E N V_{A N}$ for $B N 5=0.65 \pm 0.01 ;$ mean $\rho E N V_{A N}$ for $S S N 3=$

$2370.63 \pm 0.01)$. However, the addition of the MOC reflex increased the average $\rho E N V$ significantly for

238 Voc8 (mean $\triangle \rho E N V=+3.46 \pm 0.86 \%$, [t $(6)=10.60, p=0.0001]$ ); but reduced it significantly for

239 BN5 (mean $\triangle \rho E N V$ for BN5 $=-3.47 \pm 1.88 \%$, [t $(6)=-4.88, \mathrm{p}=0.0028]$; and also, but not

240 significantly so, for SSN3 (mean $\triangle \rho E N V$ for SSN3 $-1.42 \pm 4.01 \%, p=0.3859$ ). 


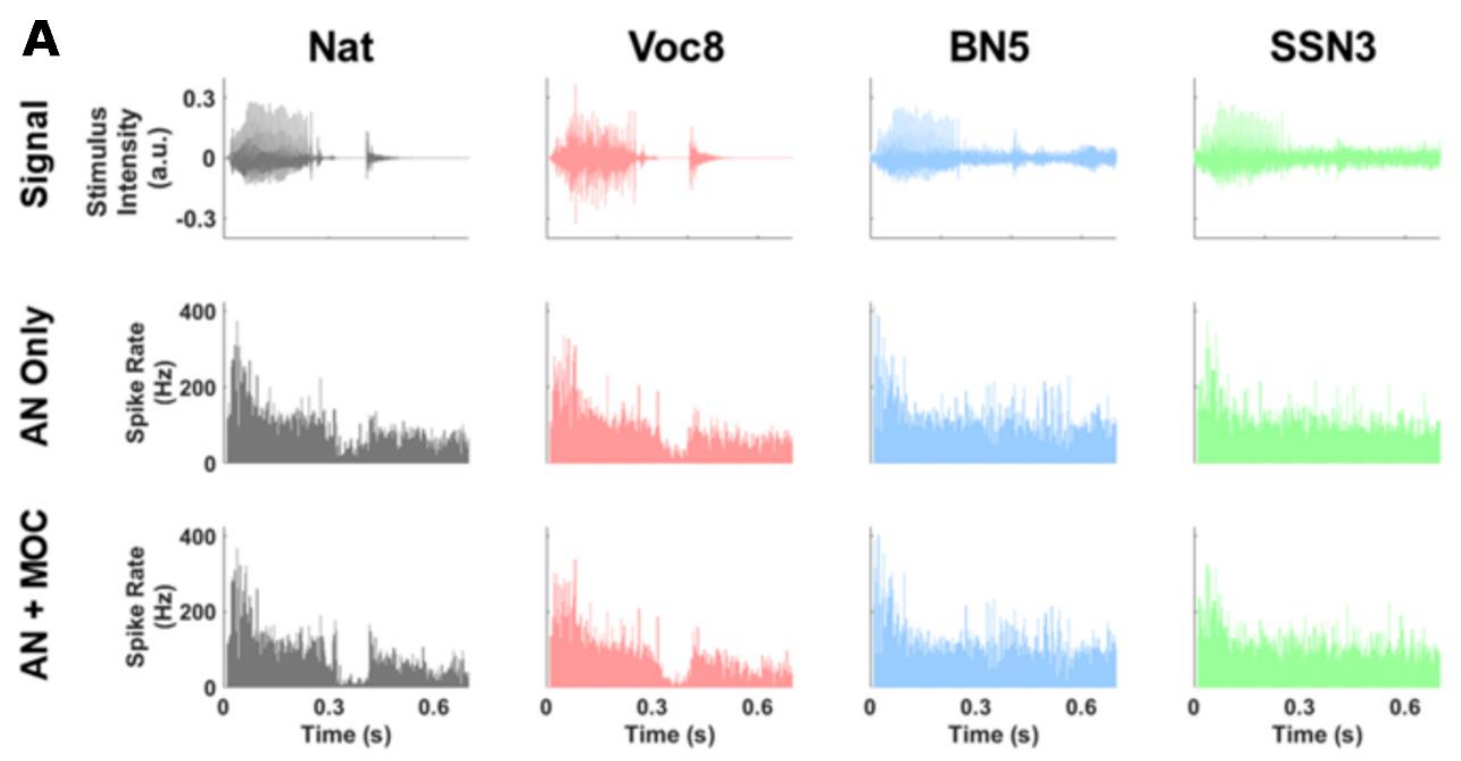

B

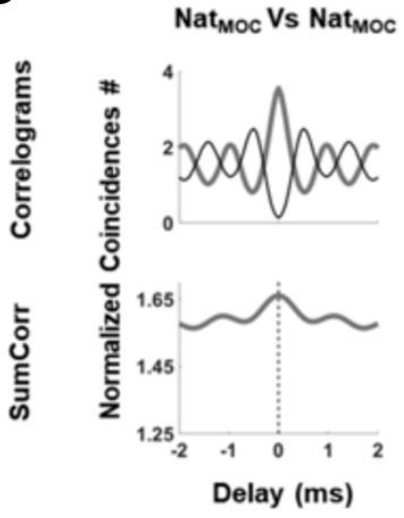

D

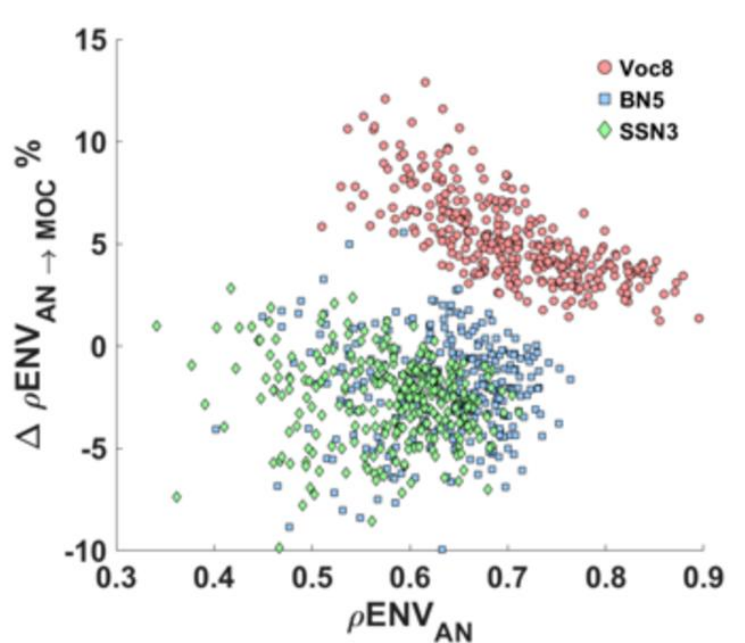

$\operatorname{Voc}_{\text {AN }}$ Vs Voc8 $8_{\text {AN }} \quad$ Nat $_{\text {MOC }}$ Vs. Voc $8_{\text {AN }}$
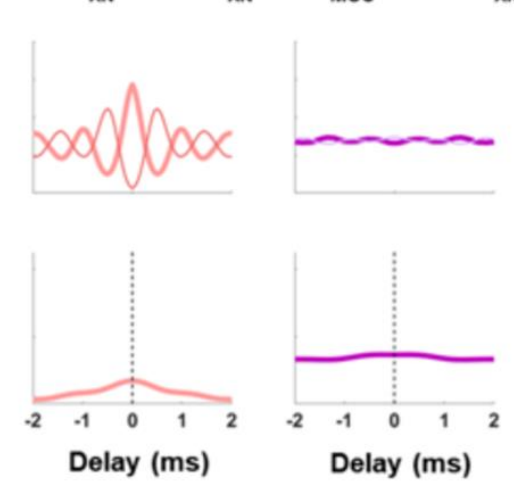

C

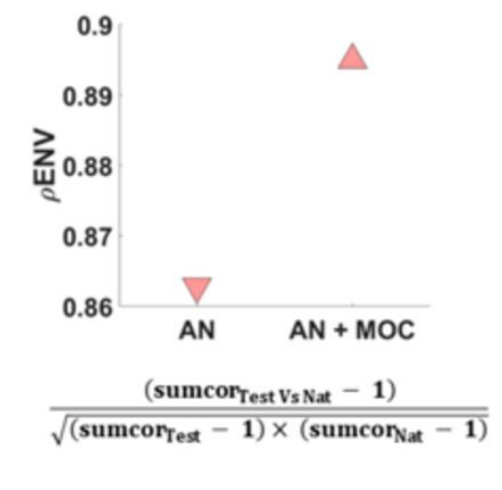

\section{E}

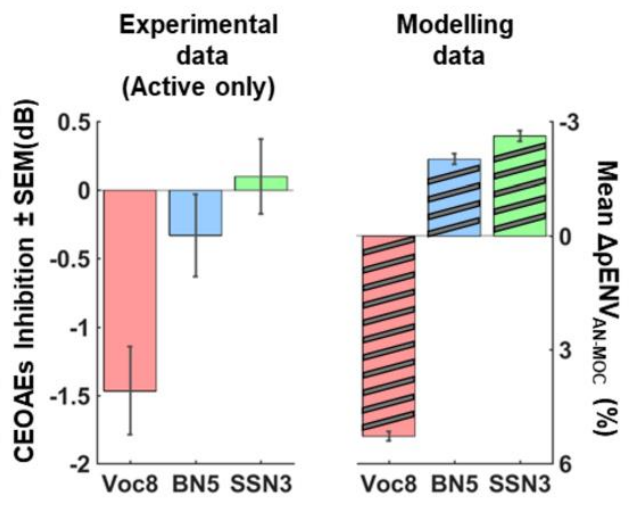

Figure 2. Model auditory periphery output with and without simulated MOC reflex. A. Presentation of natural and degraded versions of the word 'Fuzz' with and without simulated MOC reflex. 'Fuzz' waveforms for natural (dark gray, far left), Voc8 (pink, second left), BN5 (light blue, second right) and SSN3 (green, far right) conditions are shown in the top row. Post-stimulus-time-histograms (400 fibers $/ 1 \mathrm{~ms}$ bin-width) were calculated 
247 for low SR AN fibers (Characteristic Frequency: $1 \mathrm{kHz}$ ) with (bottom row) and without (middle row) simulated 248 MOC reflex. Including simulated MOC reflex reduced activity during quiet for natural condition (and Voc8, but 249 less so) whilst maintaining high spiking rates at peak sound levels (e.g. at $0.075,0.3$ and $0.45 \mathrm{~ms}$ ). No changes 250 in neural representation of signal were visually evident for BN5 and SSN3 'Fuzz'. B \& C. Quantifying $\rho E N V$ 251 for Voc8 'Fuzz' without simulated MOC reflex. Sumcor plots (bottom row, B) were generated by adding 252 Shuffled Auto-Correlograms (thick lines, top left/middle panels, B) and Cross-Correlograms (thick line, top 253 right panel, B) to cross-polarity Correlograms (thin lines, top row, B) using naturally-spoken 'Fuzz' with 254 simulated MOC reflex (left/right columns, B) and Voc8 'Fuzz' without simulated MOC reflex (middle/right 255 columns, B). $\rho E N V$ for Voc8 'Fuzz' without simulated MOC reflex (AN, downward-pointing triangle, C) was 256 calculated from SumCor peaks in B. $\rho E N V$ with simulated MOC reflex ( $A N+M O C$, upward-pointing triangle, 257 C) is also displayed. D \& E. Comparing $\triangle \rho E N V s$ for 300 words after introduction of simulated MOC reflex.

258 Mean percentage changes in $\rho E N V s$ (calculated across 7 frequencies between $1-2 \mathrm{kHz}$ ) after adding simulated $259 \mathrm{MOC}$ reflex were plotted against $\rho E N V$ without simulated MOC reflex for degraded versions of 300 words 260 (each symbol represents one word). $\triangle \rho E N V s$ were always positive for Voc8 words (pink circles, D) (Max-Min $261 \triangle \rho E N V$ for Voc8: +12.92 to $+1.24 \%$ ), appearing largest for words with lowest $\rho E N V s$ without simulated MOC 262 reflex. This relationship was absent for BN5 (light blue squares, D) and SSN3 (green diamonds, D) words 263 whose $\triangle \rho E N V$ ranges spanned the baseline (Max-Min $\triangle \rho E N V$ for BN5: +5.57 to $-9.95 \%$; Max-Min $\triangle \rho E N V$ for 264 SSN3: +2.83 to $-9.85 \%$ ). Progression of mean $\triangle \rho E N V s$ ( \pm SEM) for model data (diagonally-striped bars, right, 265 E) mirrored that of active-task, CEOAE data (mean \pm SEM) (solid-color bars, left, E). 
266 Given the range of acoustic waveforms in our speech stimuli, we expanded our analysis to include 267300 words (150 stop/non-stop consonants) (Figure 2D). Despite the diversity of speech tokens, the 268 effects of including MOC reflex (on $\rho E N V$ ) were consistently dependent on the type of stimulus 269 manipulation. Neural encoding of speech envelopes improved significantly with simulated MOC 270 reflex for all noise-vocoded words (pink circles, Figure 2D) (mean $\Delta \rho E N V$ for $V o c 8=+5.30 \pm 0.12 \%$,

$271[t(299)=42.71, p=0.0001])$, with the largest enhancements observed for words with the lowest $272 \rho E N V$ values in the absence of MOC reflex. In contrast, no such relationship was observed for 273 words in babble noise (BN5, light blue squares, Figure 2D) or speech-shaped noise (SSN3, green 274 diamonds, Figure 2D). Moreover, envelope coding in both speech-in-noise conditions was 275 significantly impaired, on average, when an MOC reflex was introduced (mean $\triangle \rho E N V$ for BN5 = $2762.01 \pm 0.14,[t(299)=-14.37, p=0.0001]$; mean $\triangle \rho E N V$ for SSN3 = -2.62 \pm 0.12 , [t $(299)=-22.03$, $277 \mathrm{p}=0.0001])$. This detrimental effect to envelope encoding with MOC reflex was significantly 278 reduced for the masked conditions with higher SNRs $(+10 \mathrm{~dB}$ SNR for babble noise and $+8 \mathrm{~dB}$ 279 SNR for speech-shaped noise) (Figure supplement 3B). In contrast, the improvement in neural 280 coding of noise-vocoded speech when introducing MOC reflex was enhanced for stimuli with more 281 noise-vocoded channels (Voc16) (Figure supplement 3B). This suggests that the MOC reflex 282 efferent feedback may specifically be unable to facilitate extraction of signal from noise at specific 283 SNRs.

284 Given recent evidence that AN fibers with high-spontaneous rate and low-thresholds (fibres that 285 respond preferentially to low intensity sounds but saturate at higher intensities [38-40] may also 286 play an important role in envelope processing [41,42], we also tested how they processed Voc8, 287 BN5 and SSN3 stimuli with and without MOC reflex. Similarly to AN fibers with high-threshold, we 288 found improvement in the envelope encoding of these low-threshold AN fibres when MOC reflex 289 was included for noise-vocoded speech, but not for the masked conditions (Figure supplement 4), 290 despite the latters' poorer dynamic range at 75 dBA (normalized sound presentation level across 291 manipulations) likely impacting their overall envelope encoding ability.

292 The pattern of enhancement of neural envelopes observed across degraded conditions when MOC 293 reflex was implemented in the model (diagonally-striped bars, Figure 2E) mirrored that observed in 
294 suppression of CEOAEs for corresponding active listening conditions (solid-colour bars, Figure 2E).

295 Where the MOC reflex was active experimentally for noise-vocoded speech, enhancement of

296 neural envelopes was observed when the same degraded stimuli were presented to the model with

297 MOC reflex present. This was not the case for active listening of masked speech - MOC reflex

298 activity being unaltered experimentally — which was associated with poorer neural representations

299 of the stimulus envelope when MOC reflex was included in the model.

300 Cortical evoked potentials reveal enhanced magnitude when active listening to speech-in-

301 noise

302 The lack of any MOC reflex contribution during active listening to speech masked by speech-like

303 sounds (i.e. babble and speech-shaped noise) compared to noise-vocoded speech suggests other

304 compensatory brain mechanisms must contribute to maintaining iso-performance with noise-

305 vocoded speech. We explored whether higher brain centres-providing top-down perhaps

306 attention-driven, enhancement of speech processing in background noise-contribute to

307 maintaining iso-performance across speech conditions. The significant increase observed in ABR

308 wave $V$ for active speech-in-noise conditions is suggestive of greater activity in the inferior colliculus

309 (IC) - the principal midbrain nucleus which receives efferent feedback from auditory cortical areas.

310 Levels of cortical engagement might then differ across speech manipulations, despite similar

311 overall task performance.

312 To determine the degree of cortical engagement in the active-listening task, we recorded cortical

313 evoked potentials simultaneously to the CEOAE and ABR measurements from all 56 participants

314 using a 64-channel, EEG-recording system. Grand averages of event-related potentials (ERPs) to

315 speech-onset (Figure 3A and Supplement Figure 5) for the most demanding speech manipulations

316 (Voc8, BN5 and SSN3) were analysed to test the hypothesis that greater cortical engagement

317 occurred when listening to speech-in-noise compared to noise-vocoded speech despite been

318 matched in task difficulty 
A
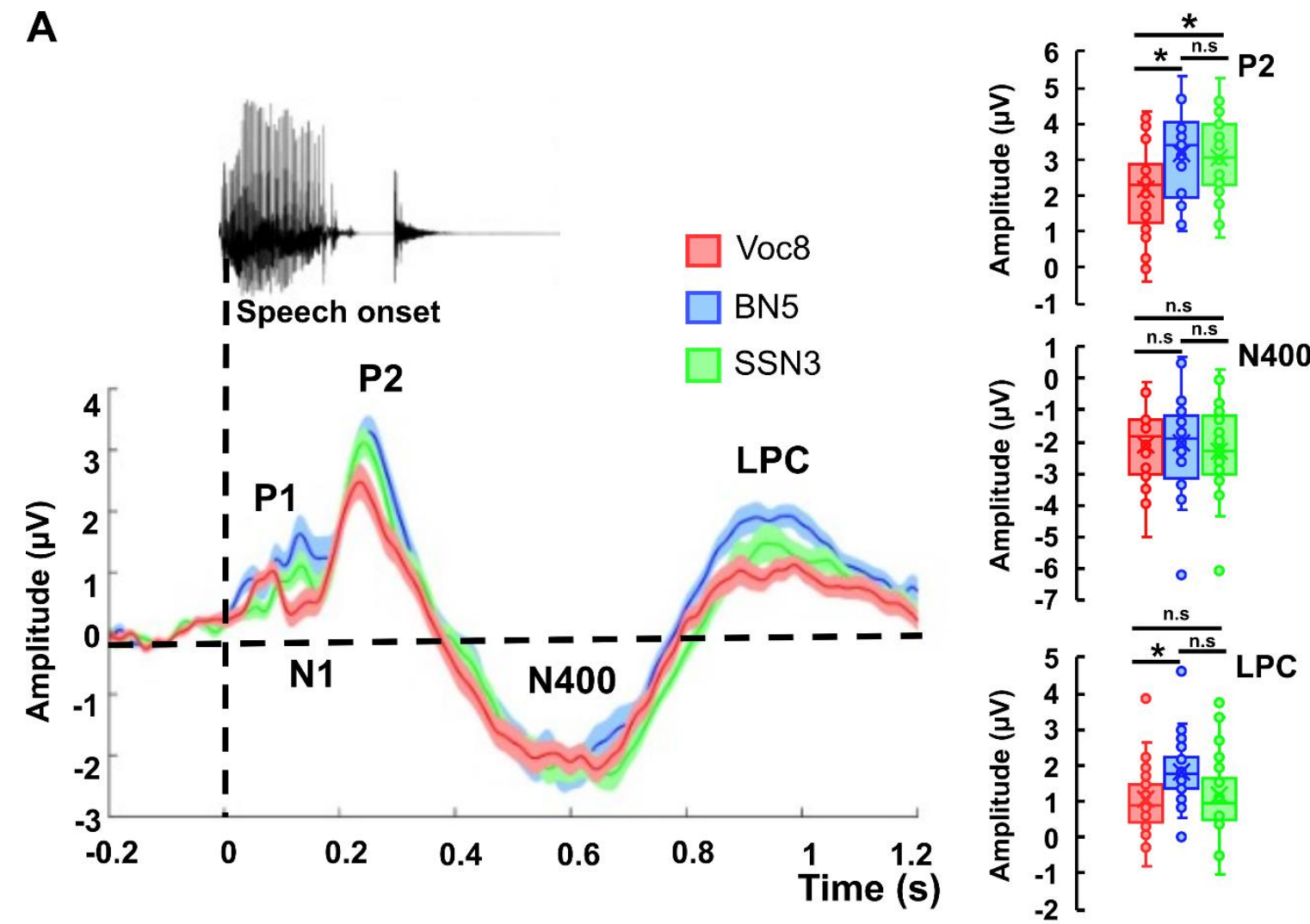

B

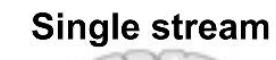

Multiple streams

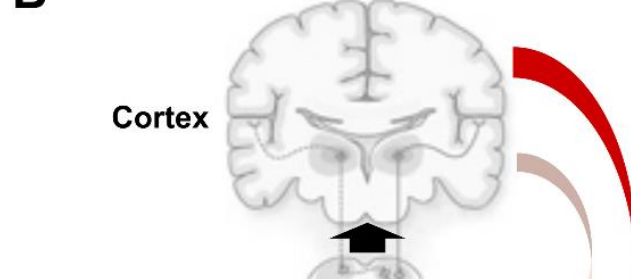

Enhanced

Suppressed
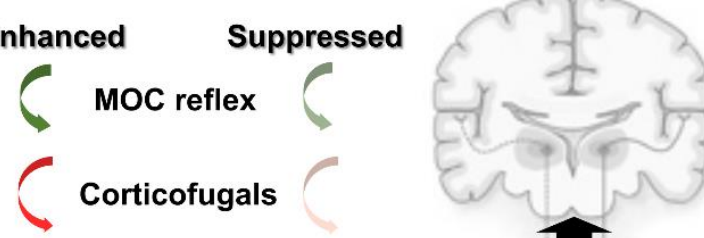

Midbrain

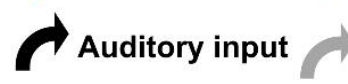

Figure 3. Cortical activity and proposed mechanisms for active listening to noise-vocoded and noise masked speech. A. Upper panel shows the "clean" natural spoken word ("Fuzz") for time-course comparisons with the ERPs. Lower panel shows ERP components (from electrodes: FZ, F3, F4, CZ, C3, C4, TP7, TP8, T7, T8, PZ, P3, P4) during the active listening of Voc8, BN5 and SSN3. Thick lines and shaded areas represent mean and the SEM. Boxplots on the right show statistical comparisons between speech conditions for P2, N400 and LPC components. B. Proposed auditory efferent mechanisms for speech processing (Auditory pathway diagrams, [43]. The "Single stream" mechanism shows how isolated degraded tokens such as noise-vocoded 
327 speech are processed in a mostly feed-forward manner (thick black arrows) (as should be the case for natural 328 speech). The activation of the MOC reflex (green arrow) improves the auditory nerve representation of speech329 envelope (auditory input) and this information passes up the auditory centres without much need to 'de-noise' 330 the signal (represented as black arrows from the cochlea-brainstem-midbrain). Given the trend for increasing 331 cochlear gain suppression with increases in task difficulty, we included the possibility for increased MOC reflex 332 drive from higher auditory regions via corticofugal connections (red arrow). In contrast, "multiple streams" such 333 as speech in babble or speech-shaped noise do not recruit the MOC reflex (shaded green arrow) because it 334 negatively affects envelope encoding of speech signals (shaded grey arrows from cochlea-brainstem335 midbrain). We therefore propose that corticofugal drive is suppressed to the MOC reflex (shaded red arrow) 336 leaving greater responsibility for speech signal extraction to the midbrain, cortex and the efferent loop therein 337 (corticofugal connections from auditory cortex to midbrain: red arrow). Both mechanisms ultimately lead to 338 equal behavioural performance across speech conditions. 
339 We analysed early auditory cortical responses (P1 and N1 components, Figure 3A) which are 340 largely driven by acoustic features of the stimulus $[44,45]$. Noise-vocoded words elicited well341 defined P1 and N1 components compared to the speech-in-noise conditions (Figure 3A). This was 342 despite words and noises having similar onsets to noise-vocoded tokens, reflecting the relatively 343 high precision of vocoded speech's envelope components at stimulus onset compared to those of 344 the masked conditions where the speech and noise envelopes overlap significantly. Later ERP 345 components, such as P2, N400 and the Late Positivity Complex (LPC), have been linked to speech346 and task-specific, top-down (context-dependent) processes [44,46].

347 Speech masked by babble or speech-shaped noise elicited significantly larger P2 components 348 during active listening compared to the noise-vocoded condition, but not significantly different 349 between themselves $[F(2,79)=5.08, p=0.008]$, post hoc with bonferroni corrections for 3 multiple 350 comparisons: [BN5 vs. Voc8 $(\mathrm{p}=0.012)$; SSN3 vs. Voc8 $(\mathrm{p}=0.041)$; BN5 vs. SSN3 $(\mathrm{p}=1.00)$ ]. 351 Similarly, the magnitude of the LPC—-thought to reflect the involvement of cognitive resources 352 including memorization, understanding [47] and post-decision closure [48] during speech 353 processing—differed significantly across conditions: $[F(2,79)=4.24, p=0.018]$. Specifically, LPCs 354 were greater during active listening to speech in babble noise compared to noise-vocoded speech $355(p=0.02)$, with LPCs obtained for active listening to speech in speech-shaped noise intermediate 356 and not significantly different to either (Figure 3A). Consistent with the concept that effortful listening 357 varied across speech-in-noise manipulations even when iso-performance was maintained $[49,50]$, 358 2016), the speech manipulation generating the clearest signature of cortical engagement was 359 speech in babble noise, considered the most difficult of the masked conditions (Figure 1B) where 360 iso-performance was achieved at $+5 \mathrm{~dB}$ SNR (BN5) compared to only $+3 \mathrm{~dB}$ SNR for speech in 361 speech-shaped-noise (SSN3). In contrast to P2 and the LPC, the N400 component of the ERP362 associated with the processing of meaning [51] —did not differ between conditions $[F(2,81)=0.22$,

$363 \mathrm{p}=0.81]$. This is unsurprising, given that participants were equally able to differentiate non-words 364 in Voc8, BN5 and SSN3 conditions, given that iso-performance had been achieved.

365 Our ERP data are consistent with differential cortical contributions to the processing of noise366 vocoded and masked speech, being larger in magnitude for speech manipulations in which the 
367 MOC reflex was less efficiently recruited i.e. babble and speech-shaped-noise, and largest overall

368 for the manipulation requiring most listening effort-speech in a background of multi-talker babble.

369 From the auditory periphery to the cortex our data suggests that two different strategies co-exist to

370 achieve similar levels of performance when listening to single undegraded/degraded streams

371 compared to masked speech (Figure 3B). The first involves enhanced sensitivity to energy

372 fluctuations through recruitment of the MOC reflex to generate a central representation of the

373 stimulus sufficient and necessary for speech intelligibility of single streams (Figure 3B, left panel).

374 The second, activated when processing speech in background noise (Figure 3B, right panel),

375 preserves cochlear gain to prevent deterioration of envelope encoding placing the onus of

376 'denoising' on midbrain and cortex including loops between them, to maximise speech

377 understanding.

378 


\section{Discussion}

380 We assessed the role of attention in modulating the contribution of the cochlear efferent system in

381 a lexical task-detecting non-words in a stream of words and non-words. Employing three speech

382 manipulations to modulate task difficulty—vocoding of words, or words masked by multi-talker

383 babble, or speech-shaped noise (i.e. noise with the same long-term spectrum as speech-we find

384 that these manipulations differentially activate the MOC reflex to modulate cochlear gain. Activation

385 of the cochlear efferent system is also dependent on whether listeners are performing the lexical

386 task (active condition) or are not required to engage whilst watching a silent, stop-motion film

387 (passive condition). Specifically, with increasing task difficulty (i.e. fewer noise-vocoded channels),

388 noise-vocoding increasingly activates the MOC reflex in active, compared to passive, listening. The

389 opposite is true for the two masked conditions, where words presented at increasingly lower SNRs

390 more strongly activate the MOC reflex during passive, compared to active, listening. By adjusting

391 parameters of the three speech manipulations - the number of noise-vocoded channels, or the

392 SNR for the speech-in-noise conditions, we find that lesser MOC reflex activity is accompanied by

393 greater magnitude of cortical activation, to maintain iso-performance in the task. A computational

394 model incorporating efferent feedback to the inner ear demonstrates that improvements in neural

395 representation of the amplitude envelope provides a rationale for either suppressing or maintaining

396 the cochlear gain during the perception of noise-vocoded speech or speech-in-noise respectively.

397 Our data suggest that a network of brainstem and higher brain circuits is involved in maintaining

398 performance in an active listening task, and that different aspects of this network, including reflexive

399 circuits in the lower brainstem, and the relative allocation of attentional resources, are differentially

400 invoked depending on specific features of the listening environment.

\section{Attentional demands reveal differential recruitment of MOC reflex}

402 Our data highlight a categorical distinction between active and passive processing of single,

403 degraded auditory streams (vocoded speech) and parsing a complex acoustic scene to hear out a

404 stream from multiple competing, spectrally-similar sounds (multi-talker babble and speech-shaped

405 noise). Specifically, task difficulty during active listening appears to modulate the cochlear gain in

406 a stimulus-specific manner. The reduction in cochlear gain with increasing task difficulty for noise- 
407 vocoded speech and, conversely, the preservation of cochlear gain when listening to speech in 408 background noise, suggests that attentional resources might gate the MOC reflex differently 409 depending on how speech is degraded. In contrast to active listening, where participants were 410 asked to ignore the auditory stimuli and direct attention to a silent film, the MOC reflex was gated

411 in a direction consistent with the auditory system suppressing irrelevant and expected auditory

412 information whilst (presumably) attending to visual streams [52-54]. Interestingly, activation of the 413 MOC reflex was observed for natural speech—further evidence that activation is not limited to tones

414 and broadband noise [55-57] —and did not depend on whether participants were required to

415 attention (i.e: were engaged in a lexical-decision task) or not. This can be explained by natural 416 speech being particularly salient as an undegraded, ethologically-relevant stimulus and the low

417 attentional load of passively watching a film resulting in the continued monitoring of unattended 418 speech [58].

419 To explain the variable reduction in cochlear gain between noise-vocoded and masked stimuli 420 across active and passive conditions, top-down and bottom-up mechanisms can be posited as 421 candidates to modify the activity of the MOC reflex. Bottom-up mechanisms, such as the increased 422 activation of the MOC reflex by wideband stimuli with flat power-spectra [59] may explain a facet of 423 our results-especially during passive listening. For instance, reduced activation of the reflex 424 observed for babble noise during passive listening may arise from being a weaker suppressor than 425 'stationary' noises such as white noise, or speech-shaped noise $[60,61]$. However, noise-vocoded 426 stimuli with relatively fewer channels might have also been thought to have activated the MOC 427 reflex more effectively due to their more 'noise-like' spectra. The absence of suppression of 428 CEOAEs in the passive noise-vocoded conditions, as well as the pattern of activation of the MOC 429 reflex across all active listening conditions, suggests that a perceptual, top-down categorization of 430 the stimuli is necessary to appropriately engage or disengage the MOC reflex.

\section{Descending control of the MOC reflex for speech stimuli is likely bilateral}

433 A central premise of our study, and those exploring the effects of attention on the MOC reflex, is 434 that otoacoustic emissions recorded in one ear can provide a direct measure of top-down 
435 modulation of cochlear gain in the opposite ear. However, it has also been suggested that activation

436 of the MOC reflex may differ between ears to expand interaural cues associated with sound

437 localization [62]. This process could be independently modulated by the extensively ipsilateral

438 corticofugal pathways described anatomically (see [63] for review). Had the activation of the MOC

439 reflex been independently controlled at either ear-for example to suppress irrelevant clicks in one

440 ear whilst preserving cochlear gain in the ear stimulated by speech-we would have expected

441 similar suppression of CEOAEs across active and passive conditions for all speech

442 manipulations - given that click stimuli were always irrelevant to the task. Instead, however, the

443 suppression of CEOAEs was both stimulus- and task-dependent, reducing the likelihood that

444 dichotic stimulus presentation engaged top-down modulation of cochlear gain differentially at either

445 ear. It remains unclear whether spatial hearing, and any role the MOC reflex plays therein, impacts

446 top-modulation given recent evidence of weaker spatial segregation cortically in tasks requiring

447 high perceptual demand (as was the case at iso-performance for both noise-vocoded and masked

448 speech conditions in our study) [64].

449 Anatomical evidence of purely ipsilateral corticofugal pathways ignores the possibility that, even

450 when presented monaurally, descending control of the MOC reflex for speech stimuli may likely be

451 bilateral. Unlike pure tones, speech activates both left and right auditory cortices even when

452 presented unilaterally to either ear [65]. In addition, cortical gating of the MOC reflex in humans

453 does not appear restricted to direct, ipsilateral descending processes that impact cortical gain

454 control in the opposite ear [66]. Rather cortical gating of the MOC reflex likely incorporates

455 polysynaptic, decussating processes that influence/modulate cochlear gain in both ears.

457 Stimulus-specific enhancement of speech envelope's neural coding explains observed 458 variability in the suppression of CEOAEs

459 Beyond extending spatial hearing [62] and the protection from noise exposure [67-69], the function

460 most commonly attributed to the activation of the MOC reflex has been its 'unmasking' of transient

461 acoustic signals in the presence of background noise $[17,55,70]$. By increasing the dynamic range

462 of mechanical $[71,72]$ and neural $[16,17]$ responses to amplitude-modulated components in the 
463 cochleae [15,73], it has been suggested that suppression of cochlear gain by the MOC reflex might

464 preferentially favour the neural representation of syllables and phonemes in speech over masking

465 noises.

466 Whilst models of the auditory periphery that include efferent feedback, typically demonstrate 467 improved word recognition for a range of masking noises (pink noise [74-76], speech-shaped noise

468 [77] and babble noise [75]), positive correlations between increased activation of the MOC reflex 469 and improved speech-in-noise perception are only sporadically reported $[23,27]$, with some studies 470 reporting negative $[22,28]$ or non-significant correlations $[29,30]$. This variability has generally been

471 attributed to methodological differences in OAE measurement (see [78] for review). Still, more 472 recent studies have questioned whether suppression of cochlear gain by the MOC reflex actually 473 benefits hearing in noise: proposing either a contribution of the reflex to perception only at specific 474 SNRs [24,78] or, in extremis, that the MOC reflex plays no role, and that neural adaptation to noise 475 statistics may instead underlay robust recognition of degraded speech $[73,79,80]$.

476 However, the stimulus- and task-dependent suppression of cochlear gain we report when speech 477 was simultaneously employed as a contralateral activator of the MOC reflex and as a target in a 478 lexical-decision task-rather than performing sequential experiments to correlate the strength of 479 the reflex as function of speech-in-noise performance, as in the majority of the reports- suggests 480 that the automatic activation of the MOC reflex is gated on or off by top-down modulation depending 481 on whether it functionally benefits or not performance. Our modelling data support this conclusion 482 by accounting for the stimulus-dependent suppression of click-evoked OAEs through enhancement 483 of speech-envelope encoding in the auditory nerve. The apparent benefit of suppressing cochlear 484 gain to neural coding of the envelope of noise-vocoded words, compared to a disbenefit for words 485 masked by background noise, is consistent with noise-vocoded words retaining relatively strong 486 envelope modulations, and that these modulations are extracted effectively through expansion of 487 the dynamic range as cochlear gain is reduced. For both noise-maskers, however, any 488 improvement to envelope coding due to dynamic range expansion applies to both speech and 489 masker, since these overlap spectrotemporally, resulting in poorer representation of the speech 490 envelope. Whilst the results of our simulations are based on average changes in the neural 
491 correlation coefficient with efferent feedback calculated for 300 words, individually, the effect of

492 suppressing cochlear gain was highly dependent on both the choice of word and the SNR at which

493 it was presented (Figure 2C and Figure supplement 3). If top-down modulation does indeed gate

494 the activation of the MOC reflex, it must account for the statistical likelihood that suppression of

495 cochlear gain can improve the neural coding of the stimulus' envelope over the duration of the task.

496 One potential criterion for predicting whether activation of the MOC reflex can improve speech

497 intelligibility is the extent to which target speech is 'glimpsed' in spectrotemporal regions least

498 affected by a background noise [81-83]. If computing speech envelope SNR in short time windows

499 is indeed key to speech intelligibility —as proposed by several models [84,85] — then this could be

500 a suitable metric by which top-down modulation of the MOC reflex could be adjusted, explaining

501 why previous studies of OAE suppression, that employ identical paradigms aside from the stimuli

502 with different spectrotemporal content (e.g: consonant-vowel pairs), can generate inconsistent

503 correlations between discrimination-in-noise and the strength of the MOC reflex $[22,86]$.

504 Additionally, changes in speech envelope SNR as a result of presenting words at different SNRs

505 should impact any benefit of activating the MOC reflex to speech intelligibility, offering an

506 explanation for correlations previously reported between the strength of the MOC reflex and task

507 performance at varying SNRs $[24,78]$.

508 In our model, switching from lowest to intermediate stimulus SNRs for both noise-maskers led to

509 an increase in the average $\triangle \rho E N V$ when efferent feedback was applied (Supplemental Figure 3B).

510 However, values of $\triangle \rho E N V$ at the intermediate SNRs were positive for only a minority of words

511 tested (73 SSN8 words and 116 BN10 words, Supplemental Figure 3A). This is consistent with the

512 lack of OAE suppression observed experimentally in the active tasks for babble noise at $10 \mathrm{~dB}$

513 SNR and speech shaped noise at $8 \mathrm{~dB}$ SNR (Figure 1C). Where an active MOC reflex does not

514 functionally benefit the neural representation of the speech envelope, it remains possible that it is

515 activated in another capacity, for example to prevent damage by prolonged exposure to loud 516 sounds [67-69].

517 Limitations of current auditory periphery model and sumcorr analysis 
518 As a stimulus feature strongly correlated with the cortical tracking and decoding of speech [87-89]

519 and the basis of several successful speech intelligibility models [84,85], the speech envelope

520 appears central to our understanding of speech. Nevertheless, the relative contributions of 521 envelope and temporal fine structure (TFS) cues to speech intelligibility remain to be determined 522 [85,90-94]. Consequently, future investigations of the functional role of the MOC reflex may benefit

523 from valid comparisons of the neural envelope and TFS representations of stimulus in response to

524 degraded speech. Such comparisons have been performed previously for noise-vocoded stimuli

525 employing a simplified model of the auditory periphery with lateral inhibition [92].

526 Quantifying sumcorr peaks served well here as a metric to correlate neural envelopes of natural 527 and differentially degraded speech. While this analysis highlighted the similarity between natural 528 and noise-vocoded speech envelopes, its results for our noise-masker simulations appear 529 counterintuitive, especially given that studies applying the same model as here, but with basic 530 efferent feedback, have previously suggested a role for the MOC reflex in speech-in-noise 531 discrimination [74-76]. These studies have employed an automatic speech recognizer (ASR) to 532 analyse AN output, encoding the shape of the speech spectrum in brief time windows to estimate 533 the speech token most likely presented as the stimulus. However, their performance reduces for 534 natural speech as efferent feedback increases [74], suggesting that this analysis would not account 535 for the experimentally observed activation of the MOC reflex for natural speech. Nevertheless, 536 further assessment of the two analyses' relative validity remains difficult, given the number of model 537 parameters that differed between this and prior studies (frequency range sampled, speech tokens 538 presented, their stimulus levels and how efferent feedback was implemented).

539 As regards the auditory periphery model itself, recent insights into the MOC reflex's circuitry will 540 enhance the biological realism of any future models incorporating efferent feedback. Our model 541 and previous phenomenological models simulate efferent feedback as a solely on-frequency effect

542 which appears unlikely given anatomical evidence that MOC neurons are sparse and innervate 543 relatively broad regions of the cochlea $[95,96]$. Additionally, most models do not include neural 544 adaptation to stimulus statistics as a potential contributing factor to speech-in-noise discrimination $545[73,79]$, we therefore cannot discount its involvement in the lexical-decision task. 
546 Higher auditory centre activity supports co-existence of multiple strategies to achieve

547 similar levels of performance

548 The impact of modulating the MOC reflex was observed in the activity of the auditory midbrain and 549 cortex. Increased midbrain activity for active noise-masked conditions was consistent with changes

550 in magnitudes of ABRs previously reported during unattended Vs attended listening to speech [97] 551 or clicks $[98,99]$. This highlights the potential for subcortical levels to either enhance attended

552 signals, or filter out distracting auditory information. At the cortical level, recorded potentials were 553 larger for all attended compared to ignored speech (Supplemental Figure 5), consistent with 554 previous reports $[100,101]$. However, later cortical components were of greater magnitude for 555 masked than noise-vocoded speech while attending to the most extreme manipulations. Late 556 cortical components have been associated with the evaluation and classification of complex stimuli 557 [46] as well as the degree of mental allocation during a task [44]. Therefore, differing cortical activity 558 likely reflects greater reliance on, or at least increased contribution from, context-dependent 559 processes for speech masked by noise than for noise-vocoded speech.

560 Taken together, the observed differences in physiological measurements from higher auditory 561 centres and the auditory periphery highlight the possibility of diverging pathways to process noise-

562 vocoded and masked speech. Evidence for systemic differences in processing single degraded

563 streams, compared to masked speech, has been reported in the autonomic nervous system [49]:

564 where, despite maintaining similar task difficulty across conditions, masked speech elicits stronger

565 physiological reactions than single unmasked streams. Here we propose that two strategies allow

566 maintaining iso-performance even when stimuli are categorically different. The processing of single

567 and intrinsically degraded streams selectively recruits auditory efferent pathways from the auditory

568 cortex to the inner ear which in turns improves the representation - 'de-noise'-of the stimuli in the

569 periphery (Figure 3B). In contrast, multiple streams appear to rely much more on higher auditory

570 centres such as midbrain and auditory cortex for the extraction of foreground, relevant signals

571 (Figure 3B). Evidence of de-noising auditory signals in the cortex [87,102-104] has led to the

572 oversight of the potential role of the extensive loops and chains of information between cortex, 
573 subcortical regions and the auditory periphery in everyday listening environments [20,105-107]

574 and therefore as target for hearing technologies.

\section{Implications for hearing-impaired listeners}

576 Although normal-hearing listeners appear to benefit from an MOC reflex that modulates cochlear 577 gain, and is amenable to top-down, attentional control, it is important to note that users of cochlear

578 implants (Cls)_for whom normal-hearing listeners processing noise vocoded speech have often

579 been a proxy-have no access to the MOC reflex. Cls bypass the mechanical processes of the

580 inner ear, including the outer hair cells-which are non-functioning or absent completely in

581 individuals with severe-to-profound hearing loss-to stimulate directly the auditory-nerve fibers

582 themselves. Efforts have been made to incorporate MOC-like properties into $\mathrm{CI}$ processes, at least

583 to provide for expanded auditory spatial cues to improve listening in bilaterally implanted $\mathrm{Cl}$ users

584 [62], but the capacity to exploit efferent feedback to aid speech understanding in $\mathrm{Cl}$ listeners is yet

585 to be exploited in any device.

586 For other hearing-impaired listeners, aided or unaided, the contribution of MOC feedback to speech

587 processing is limited compared to normal-hearing listeners as, in most cases, their hearing loss

588 comes from damage to the OHCs, which receive direct synaptic input from MOC fibers. In hearing

589 loss generally, the degradation or loss of peripheral mechanisms contributing to effective speech

590 processing in complex listening environments may mean that listeners rely more heavily on

591 attentional and other cortical-mediated processes, contributing to widely reported increases in

592 listening effort required to achieve adequate levels of listening performance [50]. This increase in

593 listening effort-likely manifesting over time-may not be reflected in performance in relatively

594 short, laboratory- or clinic-based assessments of hearing function. 


\section{Materials and Methods}

596 This study was approved by the Human Research Ethics Committee of Macquarie University (ref:

597 5201500235). Each participant signed a written informed consent form and was provided with a

598 small financial remuneration for their time.

599 Hearing assessment: All 66 participants (36 females, aged between 18-35 (mean: $24 \pm 7$ years

600 old)) included in this study had normal pure tone thresholds $(<20 \mathrm{~dB} \mathrm{HL})$; normal middle ear

601 function (standard $226 \mathrm{~Hz}$ tympanometry; normal OHCs function (assessed with Distortion Product

602 OAEs (DPOAEs) between $0.5-10 \mathrm{kHz}$. Steady-state contralateral and ipsilateral broadband noise

603 middle ear muscle reflexes (MEMR) were assessed and all participants had thresholds $>75 \mathrm{~dB}$

$604 \mathrm{HL}$.

605 Experimental Protocol: Participants were seated comfortably inside a sound-proof booth (ISO

606 8253-1:2010) while wearing an EEG cap (Neuroscan 64 channels, SynAmps2 amplifier,

607 Compumedics Limited). Two listening conditions (passive and active) were counterbalanced

608 across participants. In the passive listening condition, subjects were asked to ignore the auditory

609 stimuli and to watch a non-subtitled, stop-motion movie. To ensure participants' attention during

610 this condition, they were monitored with a video camera and were asked questions at the end of

611 this session (e.g. What happened in the movie? How many characters were present?). The aim of

612 a passive or an auditory-ignoring condition is to shift attentional resources away from the auditory

613 scene and towards the visual scene. During active listening, participants performed an auditory

614 lexical-decision task, where they were asked to press a keyboard's space key each time they heard

615 a non-word in strings of 300 speech tokens. d' was used as a measure of accuracy and calculated

616 as: Z(correct responses) - Z(false alarm).

617 Simultaneous to the presentation of word/non-word in one ear, CEOAEs were recorded 618 continuously in the contralateral ear (Figure 1A). The ear receiving either the clicks or speech 619 stimuli was randomized across participants.

620 Speech Stimuli: 423-word items were acquired from Australian-English-adapted versions of 621 monosyllabic consonant-nucleus-consonant (CNC) word lists [108] and were spoken by a female, 622 native Australian-English speaker. The duration of words ranged between 420-650 ms. 328 
623 monosyllabic CNC non-word tokens were selected from the Australian Research Council non-word 624 database [109]. Speech stimuli were delivered using ER-3C insert earphones (Etymotic Research, 625 Elk Grove Village, IL) and Presentation® software (Neurobehavioral Systems, Inc., Berkeley, CA, 626 version 18.1.03.31.15) at $44.1 \mathrm{kHz}, 16$-bits. Blocks of 200 words and 100 non-words (randomly 627 selected from the speech corpus) were presented for each of the speech manipulations (see details 628 below). Speech tokens were counterbalanced in each block based on the presence of stop and 629 non-stop initial consonants: 100 stop/ non-stop consonant words; 50 stop/non-stop consonants 630 words with a maximum of 3 repeats per participant allowed. Each block had a duration of 12 631 minutes, (Figure 1A) and participants could take short breaks between them if needed. The order 632 of blocks was randomized to prevent presentation order bias or training effects [110].

633 Noise-vocoded Speech. Twenty-seven native speakers of Australian-English (17 females: 25 634 right-handed, 2 left-handed) were recruited (aged between 18-35 (mean: $23 \pm 5$ years old). Based 635 on the noise vocoding method and behavioural results of Shannon et al. [111], three noise-vocoded 636 conditions (16-, 12- and 8-channels: Voc16, Voc12, and Voc8, respectively) were tested to 637 represent 3 degrees of speech intelligibility (i.e. task difficulty). Four blocks were assessed in both 638 active and passive listening conditions: Block 1: natural speech; Block 2: Voc16; Block 3: Voc12 639 and Block 4: Voc8, for a total duration of 2.6 hours (including hearing assessment and EEG cap 640 set-up).

641 Speech in Babble Noise. Twenty-nine native speakers of Australian-English (19 females: 28 right642 handed, 1 left-handed) were recruited, aged between 20-35 (mean: $26 \pm 9$ years old). The BN used 643 consisted of four females and four male talkers [112] and was filtered to match the long-term 644 average spectrum of the speech corpus. Random segments from 60 secs BN recording were 645 selected as noise maskers. Both speech tokens and the BN segments were matched in duration, 646 Figure supplement 6 . Three blocks were presented in the active and passive listening conditions: 647 Block1: natural speech; Block 2: BN at $+10 \mathrm{~dB}$ SNR (BN10) and Block 3: BN at $+5 \mathrm{~dB}$ SNR (BN5). 648 Speech in Speech-Shaped noise. SSN was generated to match the long-term average spectrum 649 long-term average of the speech corpus, Figure supplement 6 . Segments from 60 secs SSN were 650 randomly selected as noise maskers. Both BN and SSN manipulations were presented in the same 
651 session, therefore, Block1 was the same for both manipulations, Block 2: SSN at $+8 \mathrm{~dB}$ SNR 652 (SSN8) and Block 3: SSN at $+3 \mathrm{~dB}$ SNR (SSN3). A total of 10 blocks (5 blocks in the active and 5

653 blocks in the passive condition) of 12 min duration were presented to the 29 subjects who 654 participated in BN and SSN experiments. All blocks were presented in a unique session that lasted 6553 hours. All tokens were root-mean-square normalized and the calibration system (sound level 656 meter (B\&K G4) and microphone IEC 60711 Ear Simulator RA 0045563 (BS EN 60645-3:2007), 657 (see CEOAEs acquisition and analysis section)) was set to $75 \mathrm{~dB}$ "A-Weighting" which matches 658 the human auditory range.

659 CEOAEs acquisition and analysis. Non-filtered click stimuli, with a positive polarity and $83 \mu \mathrm{sec}$ 660 of duration were digitally generated using RecordAppX (Advanced Medical Diagnostic Systems) 661 software. The presentation rate was $32 \mathrm{~Hz}$ in all conditions which contributed to minimize ipsilateral 662 MOCR activation [113]. Ipsilateral MOCR activation was otherwise constant across participants 663 and experimental manipulations by maintaining a fixed click-rate.

664 Both the generation of clicks and OAE recordings were controlled via an RME UCX soundcard 665 (RME, Haimhausen, Germany), and delivered/collected to and from the ear canal through an 666 Etymotic ER-10B probe connected to ER-2 insert earphones with the microphone pre-amplifier 667 gain set at $20 \mathrm{~dB}$. Calibration of clicks was performed using a sound level meter (B\&K G4) and 668 microphone IEC 60711 Ear Simulator RA 0045 (BS EN 60645-3:2007). This setup was also used 669 to calibrate the speech stimuli. In addition, clicks were in-ear calibrated using forward equivalent 670 pressure level (FPL) ensuring accurate stimulus levels [114]. The OAE's probe was re-positioned, 671 re-calibrated and the block re-started if participants moved or touched it.

672 CEOAE data were analysed off-line using custom Matlab scripts (available upon request) [113].

673 The averaged RMS magnitudes of CEOAE signals were analysed between 1-2 kHz given maximal 674 MOC effects in this frequency band [115]. Only binned data for averaged CEOAEs displaying a 675 SNR $\geq 6 \mathrm{~dB}$ and with $>80 \%$ of epochs retained (i.e. had RMS levels within the two standard 676 deviations limit), were selected as valid signals for further analysis. The initial and final 60 secs of 677 CEOAE recordings of each block, in the absence of speech tokens (Figure 1A), were used as 678 baseline. As no significant differences were observed between CEOAE baseline magnitudes within 
679 participants $(p>0.05)$ across experimental conditions, all baselines were pooled within participants.

680 This allowed for an increase in both SNR and reliability of the CEOAEs individually. After baseline

681 recording, CEOAEs were continuously obtained for 10 minutes during the presentation of the

682 speech tokens. The inhibition of CEOAE magnitude relative to the baseline was calculated as

683 follows and reported as means and standard errors of the mean:

684 CEOAE inhibition $=$ CEOAE speech presentation (average across minutes) - CEOAE baseline (first 60 sec)

685 Stimuli calibration: Controlling the stimulus level is a critical step when recording any type of OAE 686 due to the potential activation of the middle ear muscle reflex (MEMR). High intensity sounds 687 delivered to an ear can evoke contractions of both the stapedius and the tensor tympani muscles 688 causing the ossicular chain to stiffen and the impedance of middle ear sound transmission to 689 increase [116,117]. As a result, retrograde middle ear transmission of OAE magnitude can be 690 reduced due to MEMR and not MOCR activation [118]. For this reason, we were particularly careful 691 to determine the presentation level of our stimuli. The presentation level for all natural, noise-

692 vocoded and speech-in-noise tokens was $75 \mathrm{dBA}$ and click stimulus at $75 \mathrm{~dB} p-p$, no MEMR 693 contribution was expected given a minimum of $10 \mathrm{~dB}$ difference between MEMR thresholds (see 694 Hearing Assessment section) and stimulus levels (ANSI S3.6-1996 standards for the conversion of $695 \mathrm{~dB}$ SPL to $\mathrm{dB}$ HL. All tokens were root-mean-square normalized calibrated using the same 696 CEOAEs calibration system (see CEOAEs acquisition and analysis).

697 EEG acquisition and analysis. EEG measurements and the CEOAE setup were synchronized 698 using a Stimtracker (https://cedrus.com/). EEG data were acquired according to the 10-20 system. 699 Impedance levels were kept below $5 \mathrm{k} \Omega$ for all electrodes. Signals were sampled at a rate of 20 $700 \mathrm{kHz}$ in the $\mathrm{AC}$ mode with a gain of 2010 and an accuracy of $0.15 \mathrm{nV} / \mathrm{LSB}$. Early and late ERP 701 components were analysed offline using Fieldtrip-based scripts [119]. Data were re-referenced to 702 the average of mastoid electrodes. Trials started $200 \mathrm{~ms}$ before and ended $1.2 \mathrm{sec}$ after speech 703 onset. Components visually identified as eye blinks and horizontal eye movement were excluded 704 from the data as well as trials with amplitude $>75 \mu \mathrm{V}$. The accepted trials $(60-80 \%$ per condition) 705 were band-pass filtered between 0.5 to $30 \mathrm{~Hz}$ with transition band roll-off of $12 \mathrm{~dB} /$ octave. Trials 706 were baseline-corrected using the mean amplitude between -200-0 ms from speech onset. 
707 Baseline-corrected trials were averaged to obtain ERP waveforms. Analysis windows centred on

708 the grand average-ERP component maximums were selected [120]: P1 (100-110 msec) and

709 N1(145-155 msec); P2 (235-265 msec), N400 (575-605 msec) and LPP (945-975 msec). Mean

710 amplitude for each component within the analysis window was calculated for each participant and

711 experimental condition.

712 EEG. Auditory Brainstem Responses (ABRs). ABR signals were extracted from central 713 electrodes (FZ, FCZ, CZ). 15 msec-duration ABR analysis windows (5 ms prior and $10 \mathrm{~ms}$ after 714 click onset) were selected. A total of 19200 trials (click rate of $32 \mathrm{~Hz}$ across 10 minutes per block) 715 were band-pass filtered between $200-3000 \mathrm{~Hz}$. Averaged ABR waveforms were obtained using a 716 weighted-averaging method [121]. Amplitude of waves III and V (Figure 1A) were visually 717 determined for each subject across blocks and conditions when appropriate (wave amplitudes 718 above the residual noise, therefore a positive SNR). Due to stimulus level restrictions $(<=75 p-p$ $719 \mathrm{~dB}$ SPL to avoid MEMR activation), wave I could not be extracted from the EEG residual noise.

720 Statistical Analysis. Sample size estimation was computed according to the statistical test 721 employed by using $G^{*}$ Power [122] (Effect size $f=0.4 ; \alpha$ err prob $=0.05$; Power $(1-\beta$ err prob) $=$ 722 0.8). All variables were tested for normality (Shapiro-Wilk test), outlier residual values preventing 723 normal distribution were removed from the data set (Table supplement 2 and 3). One-way ANOVA

724 for the behavioural and ERPs data; repeated measures ANOVAs (rANOVA) for CEOAEs and ABRs 725 data and t-tests (alpha=0.05, with bonferroni corrections for multiple comparisons) were performed.

726 One-way ANOVAs had Stimuli-Type (Natural, Voc16, BN10...etc) as Factor whereas rANOVA had 727 both Conditions (Active and Passive) and Stimuli-type as Factors. The interaction (Conditions $\mathrm{x}$ 728 Stimuli) was also explored.

729 Auditory Nerve and Brainstem Simulations. The Matlab Auditory Periphery and Brainstem 730 (MAP-BS) computational model was used to simulate auditory nerve (AN) responses with and 731 without an efferent feedback loop at seven frequencies $(1000 \mathrm{~Hz}, 1122 \mathrm{~Hz}, 1260 \mathrm{~Hz}, 1414 \mathrm{~Hz}$, $7321587 \mathrm{~Hz}, 1782 \mathrm{~Hz}, 2000 \mathrm{~Hz}$ ). A binaural brainstem circuit (Figure supplement 1A) using Hodgkin733 Huxley models was implemented to calculate within-channel efferent attenuation of the cochlear 
734 gain with biophysically realistic temporal dynamics [33] (Figure supplement 1B). The current

735 balance equation for all single-compartment, Hodgkin-Huxley models was described as follows:

736

737

738

739

740

741

742

743

744

745

746

747

748

750

751

752

753

754

755

756

757

758

759

760

761

762

$$
\begin{aligned}
C_{m} \frac{d V}{d t}= & -I_{N a}-I_{K L T}-I_{K H T}-I_{H}-I_{L}-I_{s y n}(t) \\
= & -\bar{g}_{N a} m^{3} h\left(V-E_{N a}\right)-\bar{g}_{K L T} w\left(V-E_{K}\right)-\bar{g}_{K H T} n\left(V-E_{K}\right)-\bar{g}_{H} r\left(V-E_{H}\right)-\bar{g}_{L}\left(V-E_{L}\right) \\
& \quad-A \bar{g}_{s y n}(t)\left(V-E_{s y n}\right)
\end{aligned}
$$

Where $\bar{g}_{x}$ is the maximum channel conductance and $E_{x}$ is the corresponding reversal potential for conductance $x$ where $x$ was either: $N a$ (fast sodium), KLT (low threshold potassium), KHT (high threshold potassium), $H$ (hyperpolarization-activated mixed-cation), $L$ (leak) and $K$ (all potassium conductances) (see Table supplement 1 for relative values in cell types). $\bar{g}_{s y n}(t)$ is the unitary synaptic input at time, $t$, and $A$ is an input activity-dependent factor with a predetermined, maximum value for each cell type (Max Synaptic Current, Table supplement 1). $E_{\text {syn }}$ represents the synaptic reversal potential which was either $0 \mathrm{mV}$ for excitation or $-70 \mathrm{mV}$ for inhibition (Figure supplement $1 \mathrm{~A}$ and Table supplement 1). An absolute voltage threshold of $-20 \mathrm{mV}$ was used for suprathreshold responses. The dynamics of gating variables $(m, h, w, n$ and $r$ ) were described by the following differential equation:

$$
\frac{d x}{d t}=\frac{x_{\infty}(V)-x}{\tau_{x}(V)}
$$

Where $\operatorname{tx}(V)$ is the time constant of the variable $x$ and $x^{\infty}(V)$ is the steady state value of $x$ at voltage, $V$. See 'HH5lookuplnt.m' file in MAP-BS [33] for values of $T x(V)$ and $x^{\infty}(V)$ for individual conductances. The spiking output of MOC neurons was used to calculate inhibition of the basilar membrane displacement's nonlinear path [75] as follows:

$$
\operatorname{ATT}(t)=\left[1+M O C_{1}(t)+M O C_{2}(t)\right]^{-1}
$$

Where the formula for calculating activation and exponential decay of $M O C_{x}$ at time, $t$, was expressed as:

$$
\operatorname{MOC}_{x}(t)=\left[\left(M O C_{x}(t-1) \times \exp ^{-d t / \tau_{x}}\right)+\left(F w_{x} \times \operatorname{Spikes}_{l / r}(t-t d)\right)\right]
$$

Where $\mathrm{T}_{\mathrm{x}}$ is the decay time constant (55ms for $M O C_{1}$ and $400 \mathrm{~ms}$ for $M O C_{2}$ (Backus and Guinan, 2006) and Spikes(t-delay) is spikes observed across 'left' and 'right' MOC populations at a time, $t$ - 
$763 t d$ (where $t d$ equals a $10 \mathrm{~ms}$ lag associated with synaptic latencies from MOC fibers to OHCs [123].

$764 \quad F$ is a temperature- and sample rate-dependent factor attenuating Spikes(t-delay) appropriately

765 (0.02 here for a default temperature of $38 \mathrm{C}$ and a sample rate of $100 \mathrm{kHz}$ ). $w_{x}$ is a weighting factor

766 determining the relative contribution of $\mathrm{MOC}_{1}$ and $\mathrm{MOC}_{2}$ to attenuation $\left(0.9\right.$ and 0.1 for $\mathrm{MOC}_{1}$ and

$767 \quad \mathrm{MOC}_{2}$ respectively).

768 Word Presentation. 300 words (150 stop/non-stop consonant words), were chosen at random 769 from the speech corpus and were degraded using the most demanding speech manipulations 770 (Voc8, BN5 and SSN3). Normal, Test+ and polarity-inverted, Test-, versions of each manipulation 771 were presented to the MAP-BS model at $75 \mathrm{~dB}$ SPL both with and without efferent feedback. 772 Natural words (both normal, Nat+, and polarity-inverted, Nat-) were also presented to the MAP-BS 773 model with efferent feedback enabled as a reference AN output (Figure supplement 2).

774 Shuffled Auto-Correlogram Analysis. Comparative analysis of AN coding of amplitude775 modulated (AM) envelope between Voc8/BN5/SSN3 conditions and the reference natural condition 776 was performed using shuffled auto- and cross- correlograms (SACs and SCCs respectively)[124] 777 Normalized all-order histograms were calculated using the spike trains of 400 low SR, AN fibers 778 with a coincidence window of $50 \mu$ s and a delay window $\pm 25 \mathrm{~ms}$ centred on zero [37]. No correction 779 for triangular shape was required given brevity of delay window relative to stimulus length (between $780420-650 \mathrm{~ms}$ ) [124]. A neural cross-correlation coefficient, $\rho E N V$, quantifying AM envelope encoding 781 similarity between conditions was generated as follows [37]: Where sumcorNat (natural word reference) and sumcorTest (Voc/BN/SSN conditions) are the 787 /sumcorTest respectively) and cross-polarity histograms (Normalized all-order histograms for Nat+ 788 vs. Nat-/Test+vs.Test- for sumcor ${ }_{N a t} /$ sumcorTest respectively). SumcorTest Nat is the average of the 789 SCC (Average of normalized all-order histograms for Nat+ vs. Test+ and Nat- vs. Test-) and the 790 cross-polarity correlogram (Average of normalized all-order histograms for Nat- vs. Test+ and Nat+ 791 vs. Test-) between natural and Voc8/BN5/SSN3 conditions. All high-frequency oscillations (> test- 
792 frequency), associated with fine-structure leakage, were removed from sumcors (Heinz and

793 Swaminathan, 2009). $\rho E N V$ values ranged from 0 to 1 where 0 represents completely dissimilar

794 spike trains and 1 represents identical spike patterns [37].

795 Analysis of modelling and statistics. Percentage changes in $\rho E N V$ due to efferent feedback

796 inclusion in MAP-BS were calculated for each test frequency and Voc8/BN5/SSN3 condition as

797 follows:

798

799

800

801 Where $\triangle \rho E N V_{\text {freq }}$ is the percentage change in $\rho E N V$ at a test-frequency for a manipulated word.

$802 \rho E N V_{\text {no eff }}$ and $\rho E N V_{\text {eff }}$ are measures of $\rho E N V$ with and without efferent feedback enabled

803 respectively. An average $\Delta \rho E N V_{\text {freq }}$ was calculated across test-frequencies for each word and

804 manipulation. A one-sample t-test was then performed to confirm whether average $\Delta \rho E N V_{\text {freq }}$ for

805 all words differed from zero for each speech manipulation. Data are reported as means and 806 standard errors of the mean. 
bioRxiv preprint doi: https://doi.org/10.1101/2020.05.31.115444; this version posted March 19, 2021. The copyright holder for this preprint (which was not certified by peer review) is the author/funder. All rights reserved. No reuse allowed without permission.

\section{Acknowledgements}

808

809 H.H.P. was supported in this study by an International Macquarie University Excellence Scholarship 810 and the Hearing CRC. The authors thank Prof. David Poeppel for his contributions during 811 experimental design. We thank Prof. David Ryugo for his comments on the manuscript. In addition, 812 we thank Ronny Ibrahim, Jaime Undurraga and Greg Stewart for their technical support regarding 813 equipment and EEG analysis; Andrew Brughera for his help with figures and Nicholas Clark for his 814 assistance with MAP-BS.

815

816

817

818 The author has no financial interests to declare. The other authors declare that no competing 819 interests exist. 

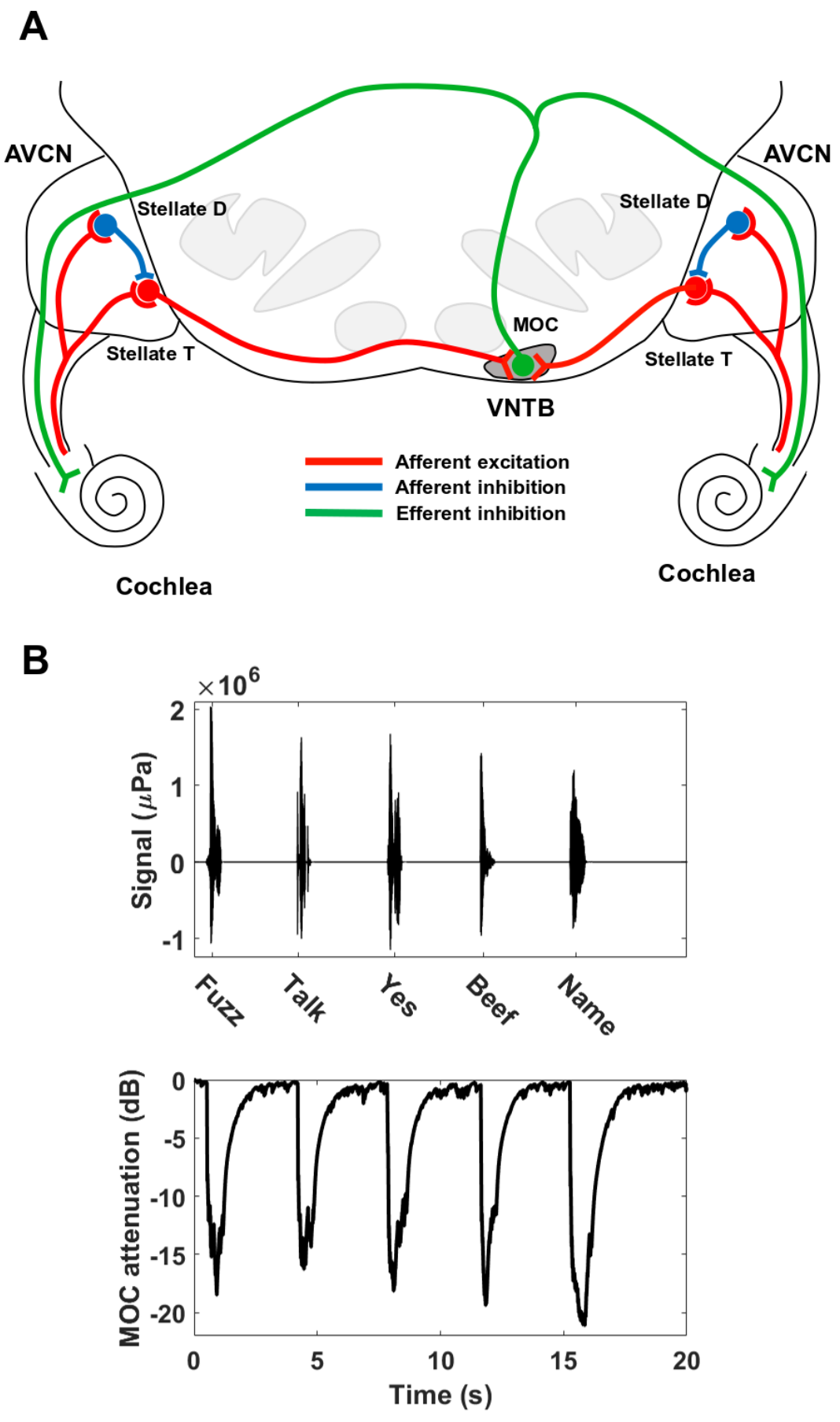

822 Figure supplement 1. Brainstem circuit and MOC attenuation of MAP-BS. A. Overview of brainstem circuit in MAP-BS. Bilateral afferent excitation (red lines) via high spontaneous rate auditory nerve (AN) fibers drives Stellate T (within-channel AN input) and D neurons (wideband AN input) in anteroventral cochlear nucleus (AVCN). Stellate D neurons provide ipsilateral, feedforward inhibition to Stellate T neurons in the same frequency channel (blue lines). Ventral nucleus of the trapezoid body (VNTB) neurons receive bilateral excitation from Stellate T neurons; their output is combined across 'left' and 'right' brainstems to calculate within-channel, efferent attenuation to both cochleae (green lines). Emulating inhibition of cochlear amplification, VNTB output impacts the non-linear pathway of dual-resonance nonlinear model of cochleae 
bioRxiv preprint doi: https://doi.org/10.1101/2020.05.31.115444; this version posted March 19, 2021. The copyright holder for this preprint (which was not certified by peer review) is the author/funder. All rights reserved. No reuse allowed without permission.

830 with a short delay [75,123]. B. Temporal dynamics of efferent feedback in MAP-BS. Upper panel shows 831 waveforms of naturally-spoken word tokens presented diotically at $75 \mathrm{~dB}$ to MAP-BS model with a 3 sec pause 832 between tokens as in the psychoacoustic paradigm (Figure 1A). Lower panel presents MOC attenuation 833 calculated across 'left' and 'right' VNTB populations. MOC attenuation did not summate across series of word 834 tokens but recovered to baseline; single-shot presentation of word tokens was therefore implemented for the 835 main results. 
bioRxiv preprint doi: https://doi.org/10.1101/2020.05.31.115444; this version posted March 19, 2021. The copyright holder for this preprint (which was not certified by peer review) is the author/funder. All rights reserved. No reuse allowed without permission.

A
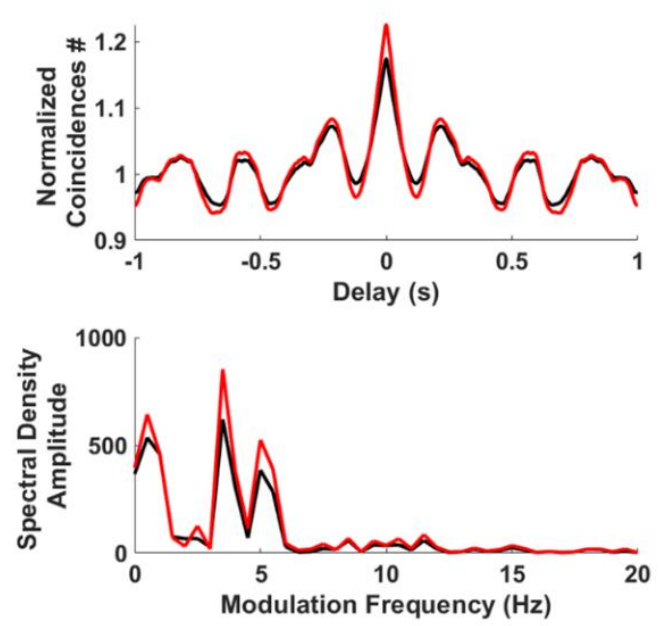

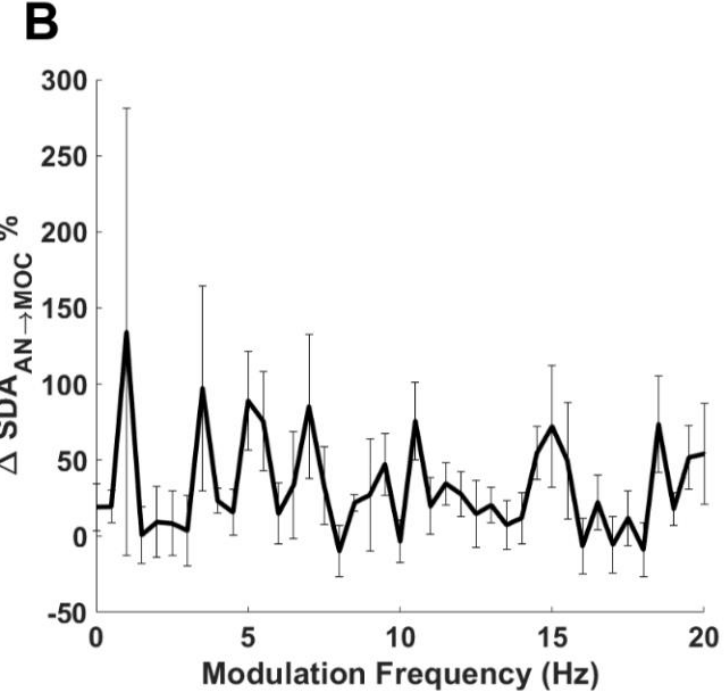

836

837

838

839

840

841

842

843

844

845

846

847

848

849

Figure supplement 2. Efferent feedback improves envelope encoding for naturally-spoken sentences. A. Shuffled-Correlogram Sumcors (upper panel) were calculated for the naturally-spoken sentence [125], 'the steady drip is worse than the drenching rain' (s86; The MAVA corpus [126]), using low spontaneous rate auditory nerve fiber output between $1-2 \mathrm{kHz}$ with (red line) and without (black line) efferent feedback. A longer, $1 \mathrm{sec}$ delay-window was used compared to the single word presentation; in addition, inverted triangular compensation was implemented to compensate for large delays relative to signal length (Rallapalli and Heinz 2016). The envelope power spectral density (lower panel) was computed both with (red line) and without (black line) efferent feedback by computing Fourier transforms of the above Sumcors with a $<1 \mathrm{~Hz}$ spectral resolution. Efferent feedback was conducive to larger envelope responses, especially at low modulation frequencies associated with words and syllables. B. Mean percentage change ( \pm SEM) in power spectral density amplitude $\left(\triangle S_{S D A N \rightarrow M O C}\right)$ with efferent feedback included was computed using 6 sentences (s7, s26, s37, s42, s86, s164; The MAVA corpus) for modulation frequencies below $20 \mathrm{~Hz}$. As for the single example in $A$, adding efferent feedback improved envelope encoding across most modulation frequencies. 


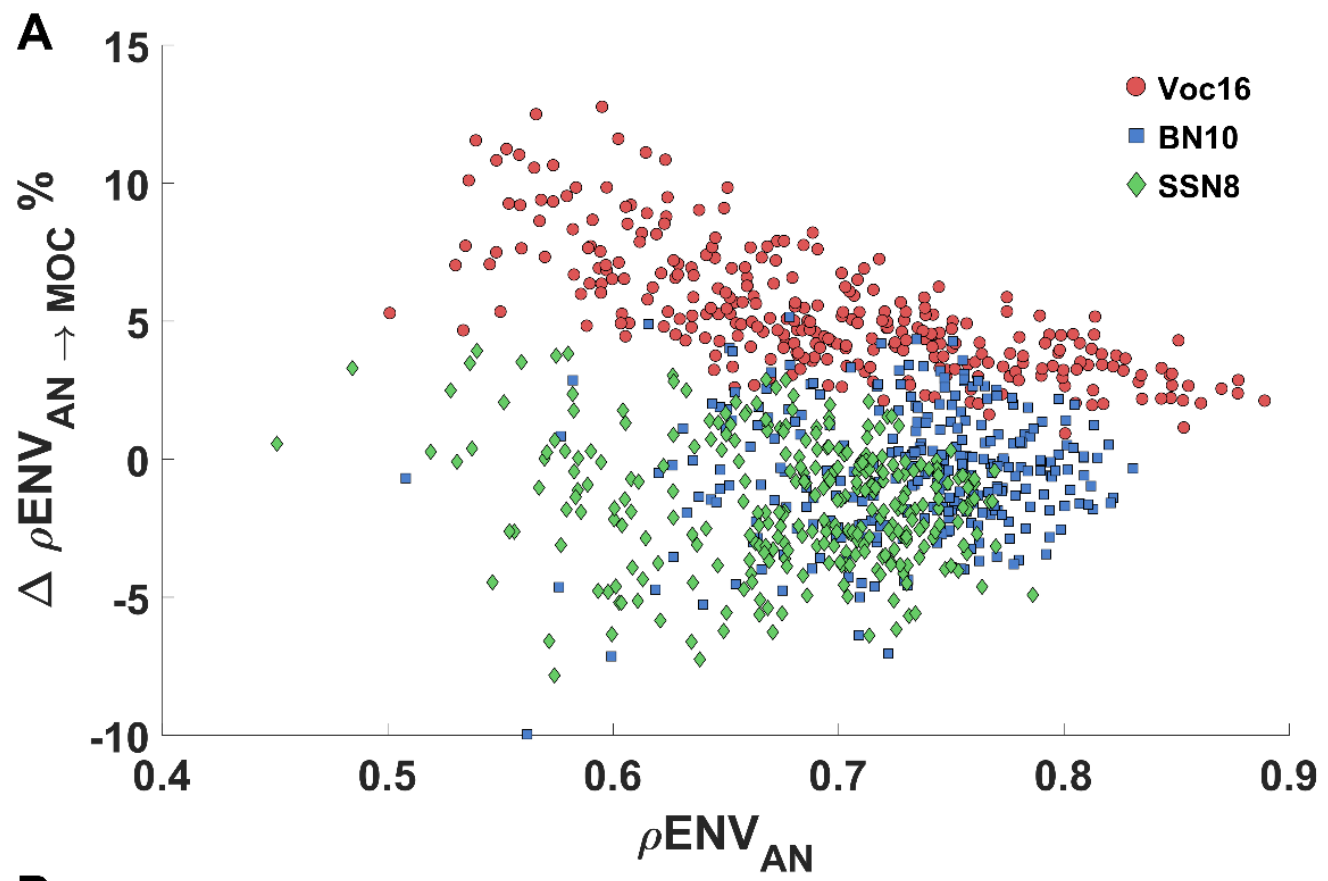

B
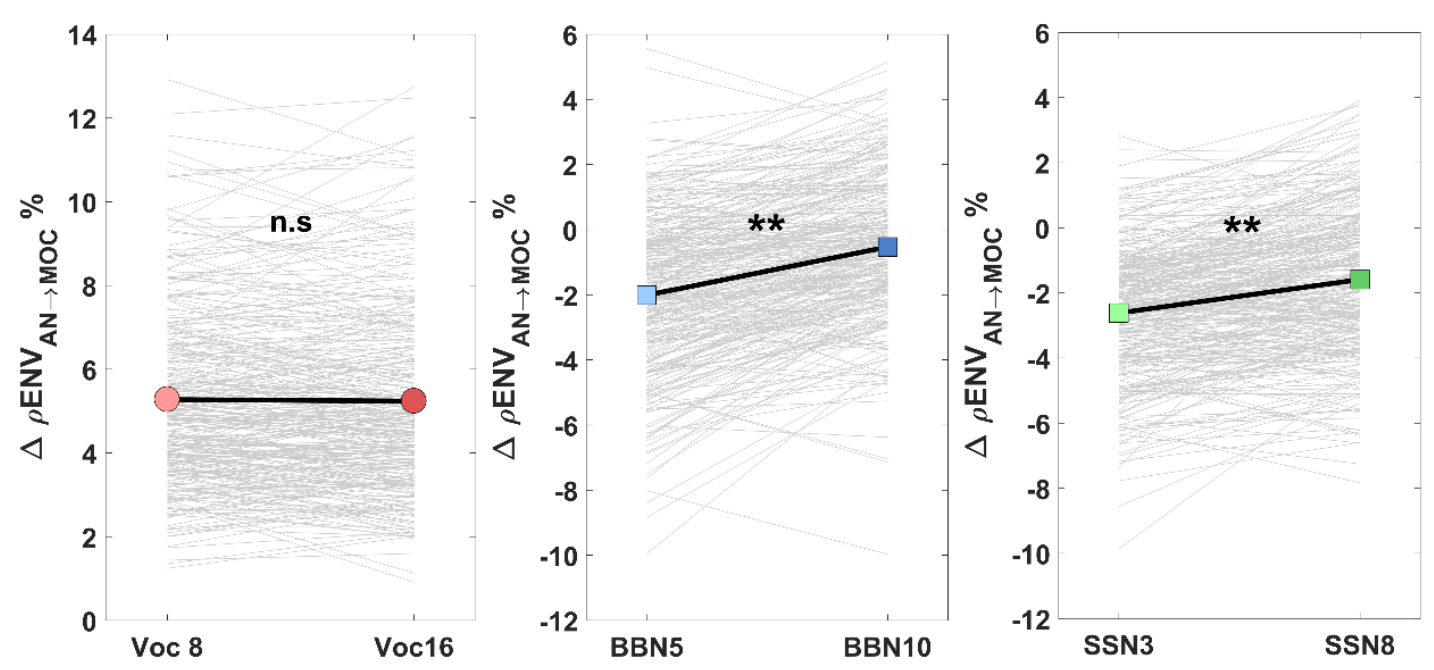

Figure supplement 3. Presentation of degraded speech tokens with higher SNRs/more channels. A. Comparing $\triangle \rho E N V s$ for 300 words after introduction of efferent feedback for Voc16, BBN10 and SSN8 stimuli. Mean percentage changes in $\rho E N V s$ (calculated across 7 frequencies between $1-2 \mathrm{kHz}$ ) after adding efferent feedback were plotted against $\rho E N V$ without efferent feedback for degraded versions of 300 words (each symbol represents one word). $\triangle \rho E N V s$ were always positive for Voc16 words (pink circles, D) (Max-Min $\triangle \rho E N V$ for Voc8: +12.75 to $+0.91 \%$ ), appearing largest for words with lowest $\rho E N V$ s without efferent feedback. This relationship was absent for BN5 (light blue squares, D) and SSN3 (green diamonds, D) words whose $\triangle \rho E N V$ ranges spanned the baseline as they did for BN and SSN stimuli with lower SNRs (Max-Min $\triangle \rho E N V$ for BN10: +5.16 to $-9.97 \%$; Max-Min $\triangle \rho E N V$ for SSN8: +3.92 to $-7.83 \%)$. B. Mean $\Delta \rho E N V$ for BN10 and SSN8, however, increased significantly compared to their lower SNR counterparts, BN5 and SSN3 (mean $\triangle \rho E N V$ for BN5 Vs BN10 = -2.01 \pm 0.14 Vs. $-0.5294 \pm 0.13[t(299)=-15.32, p=0.0001]$; mean $\triangle \rho E N V$ for SSN3 Vs SSN8 $=-2.62 \pm 0.12$ Vs. $-1.59 \pm 0.13$, $[\mathrm{t}(299)=-10.89, p=0.0001])$. Mean $\Delta \rho E N V$ for Voc16, on 863 the other hand, was not significantly different from the Voc8 condition (mean $\triangle \rho E N V$ for Voc8 Vs Voc 16= $864+5.30 \pm 0.12 \%$ Vs. $+5.24 \pm 0.13 \%$, $[\mathrm{t}(299)=0.6535, p=0.51])$. 


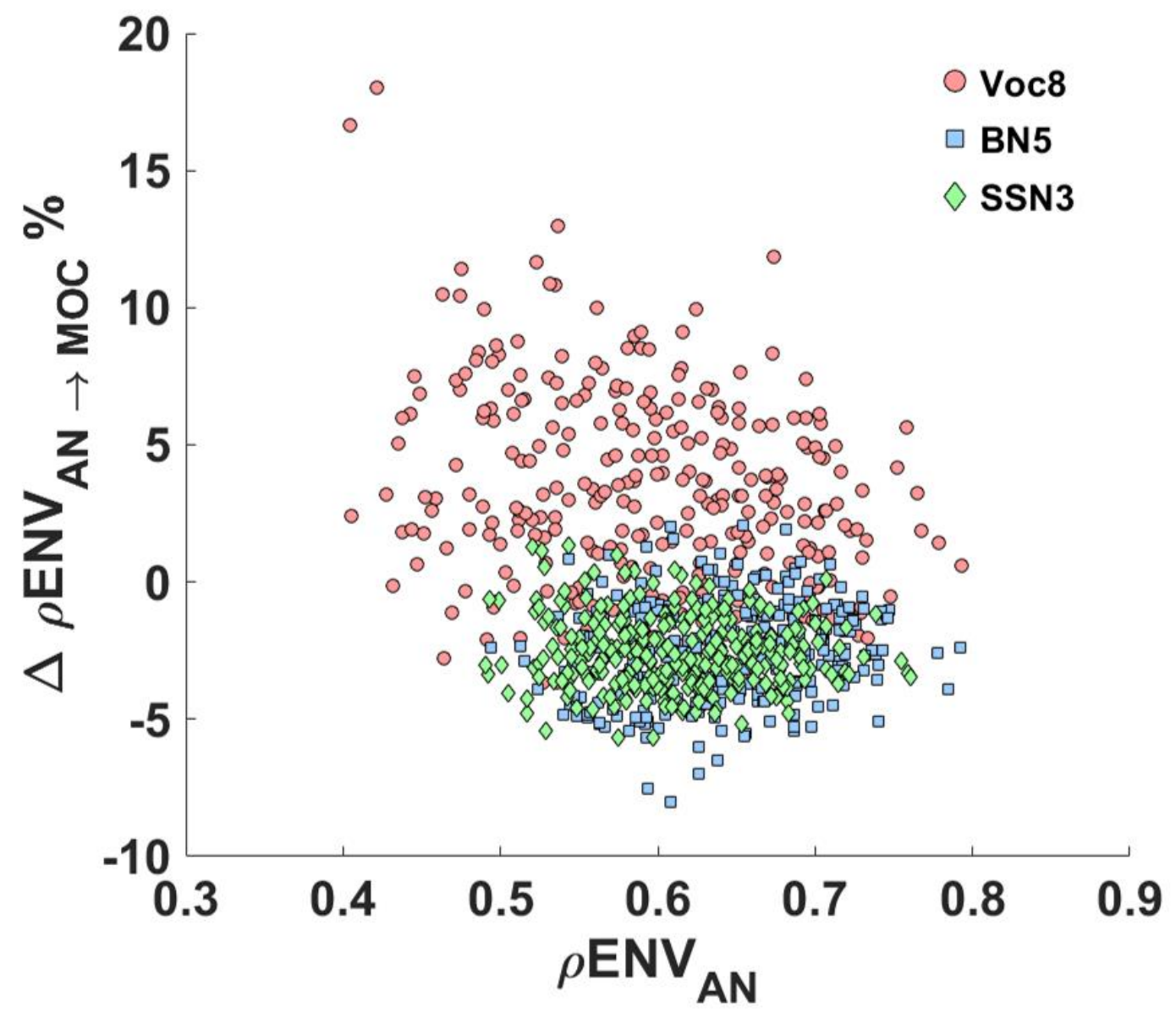

865 Figure supplement 4. Percentage change in envelope encoding of high spontaneous rate (HSR) auditory 866 nerve (AN) fibers after introduction of efferent feedback. $\triangle \rho E N V s$ for 300 words (in their three degraded forms) 867 were calculated as in Figure 2D; however, HSR AN fibre output was used here. Although $\triangle \rho E N V s$ for Voc8 868 words (pink circles) varied greatly and included negative values (Max-Min $\Delta \rho E N V$ for Voc8 $=+16.87$ to $8691.94 \%$ ), mean $\Delta \rho E N V$ was significantly positive (mean $\Delta \rho E N V$ for $\operatorname{Voc} 8=+5.42 \pm 0.18 \%$, [t $(299)=30.17, p$ $870=0.0001])$. Note that values of $\rho E N V_{A N}$ for Voc8 were significantly smaller here compared to matched values 871 for low SR (LSR) AN fibers (mean $\rho E N V_{\text {AN-HSR }}$ for Voc8 $=0.58 \pm 0.01$ vs. mean $\rho E N V_{\text {AN-LSR }}$ for Voc8 $=0.70 \pm$ $8720.01,[\mathrm{t}(299)=-39.21, \mathrm{p}=0.0001]$ ). In contrast, the distributions of $\triangle \rho E N V s$ for BN5 (light blue squares) and 873 SSN3 (green diamonds) appeared more compact (Max-Min Range $\triangle \rho E N V$ for BN5 words $=+2.06$ to $-8.04 \%$; 874 Max-Min $\triangle \rho E N V$ for SSN3 = +1.32 to $-5.67 \%$ ); however, as for LSR AN fibre results, both mean $\Delta \rho E N V s$ for 875 HSR AN fibers were significantly negative overall (mean $\triangle \rho E N V$ for BN5 $=-2.54 \pm 0.10$, [t(299) $=-24.92, p=$ 876 0.0001]; mean $\triangle \rho E N V$ for SSN3 $=-2.50 \pm 0.08,[t(299)=-32.64, p=0.0001])$. 


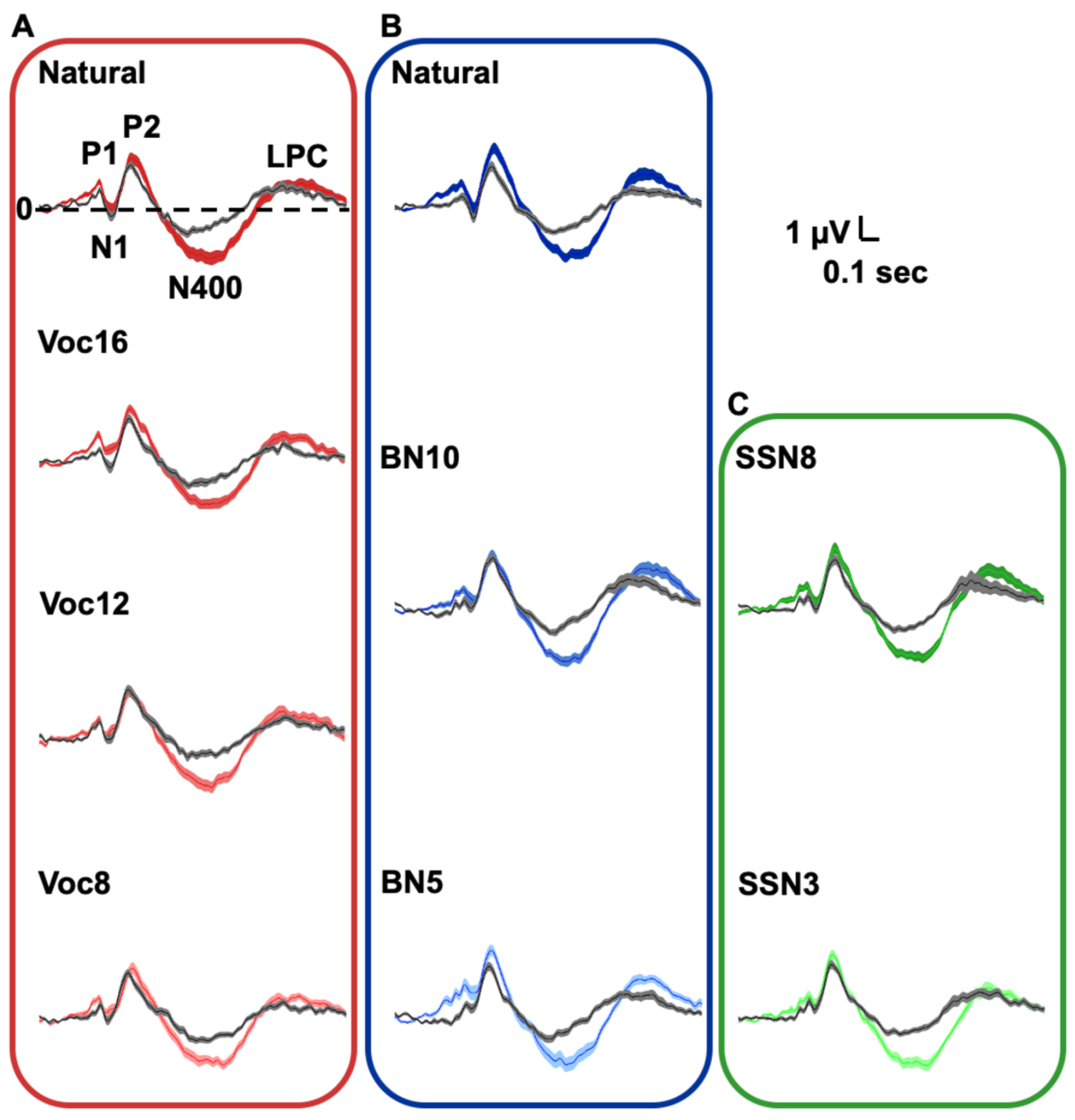

A

Figure supplement 5. Cortical evoked potentials during active and passive speech perception. Thick lines and shaded areas represent means and standard errors of the mean respectively. Within conditions analysis showed that, for all speech manipulations, the magnitude of $\mathrm{P} 1, \mathrm{P} 2$ and N400 potentials were enhanced during active (color lines) when compared to the passive (gray lines) listening conditions, whilst $\mathrm{N} 1$ tended to be less negative in the active task. Late Positivity Complex (LPC) magnitude was only significantly enhanced during the active listening of speech in noise. A. ERP components in natural and all noise-vocoded manipulations: $P 1:[F(1,24)=6.36, p=0.02], N 1:[F(1,24)=16.03, p=0.001], P 2:[F(1,24)=12.30, p=0.002], N 400:[F$ $(1,24)=31.82, p=0.0001]$, LPC: $[F(1,24)=5.29, p=0.03]$. B. ERPs during natural (different population than noise-vocoded experiment) and all babble noise manipulations $(n=29)$ : $P 1:[F(1,28)=24.47, p=0.0001]$, $N 1:[F(1,28)=10.46, p=0.003], P 2:[F(1,28)=10.65, p=0.003], N 400:[F(1,28)=62.16, p=0.0001$, LPC: $[F(1,28)=10.55, p=0.003]$. C. ERP components during speech-shaped noise manipulations $(n=29)$ : $P 1$ : $[F(1,28)=22.98, p=0.0001], N 1:[F(1,28)=6.07, p=0.02], P 2:[F(1,28)=18.10, p=0.001]$ and $N 400$ : $[F(1,28)=60.75, p=0.0001])$, LPC: $[F(1,28)=10.76, p=0.003]$ 


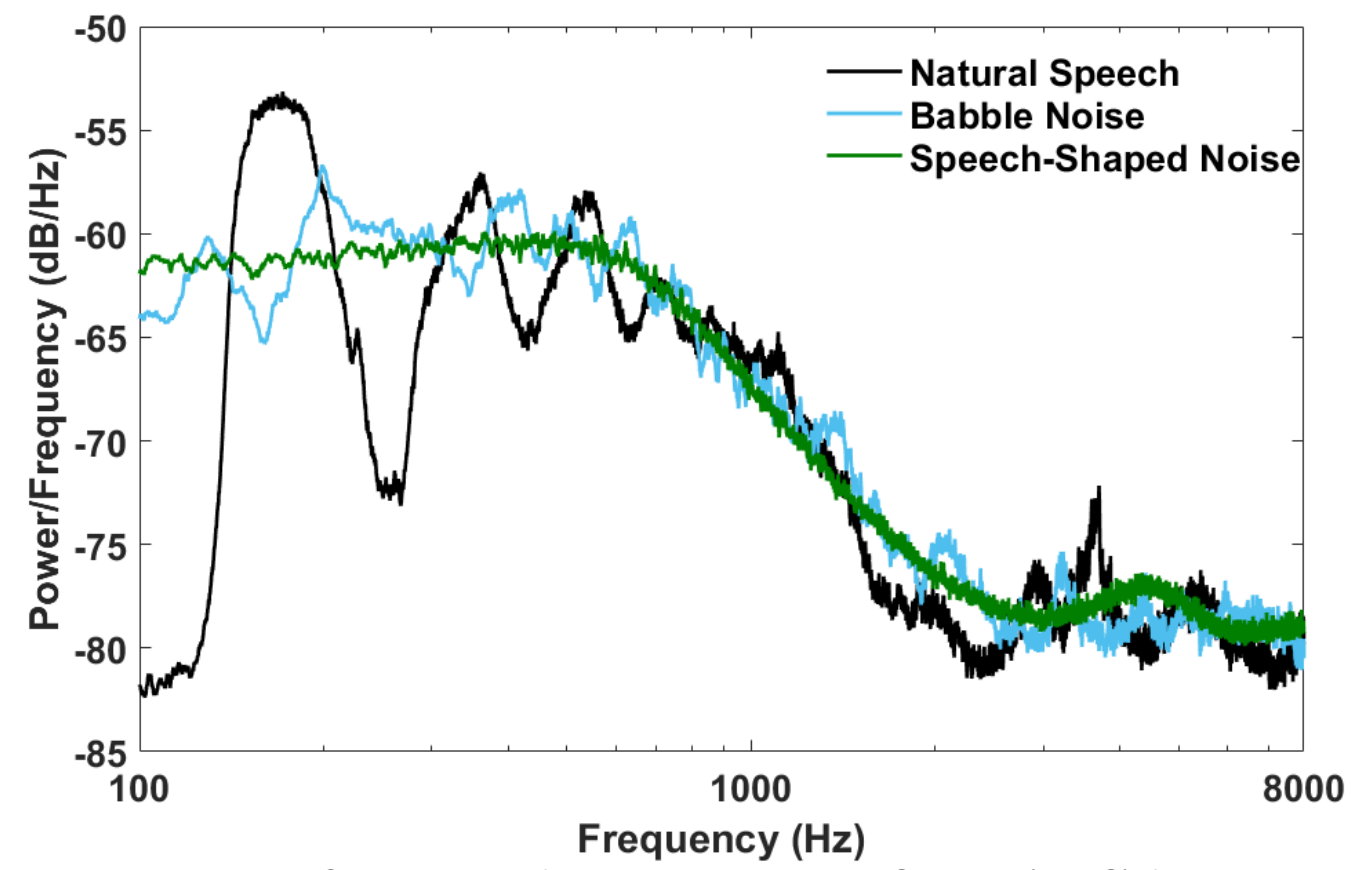

891

892

893

894

895

896

897

Figure supplement 6. Comparison of Long-Term Average Spectra (LTAS) for natural speech, babble and speech-shaped noises. Power spectrum density estimates were calculated for 300 concatenated natural speech tokens (those presented to the MAP_BS model) and 60 seconds of 8-talker babble noise and speech-shaped noise; all acoustic stimuli had been normalised to $65 \mathrm{~dB}$. The upper root-mean square envelopes, generated using 300-point sliding windows, are shown for the different conditions. 
Table supplement 1. Hodgkin-Huxley model parameters and synaptic properties

\begin{tabular}{|c|c|c|c|c|c|}
\hline & LSR AN Fiber & HSR AN Fiber & T Stellate & D Stellate & VNTB \\
\hline Cell number & 200/CF/ear & 200/CF/ear & 50/CF/side & 50/CF/side & 50/CF/side \\
\hline Cell type inputs: & ['ipsi' LSR syn] & ['ipsi' HR syn] & ['ipsi' AN (HSR); 'ipsi' VCN(chD)] & ['ipsi' AN(HSR)] & ['contra' VCN (ChT); 'ipsi' VCN (ChT) \\
\hline Input Bandwidth (Octaves) & {$[\mathrm{CF}]$} & [CF] & [CF; CF] & [2] & [CF; CF] \\
\hline Number of inputs & [1] & [1] & {$[15 ; 5]$} & [25] & {$[20 ; 5]$} \\
\hline Max Synaptic Current (nA) & [30] & [40] & {$[2 ; 10]$} & [20] & {$[3 ; 3]$} \\
\hline Synaptic Delay (ms) & [1.1] & [1.1] & {$[1.9 ; 1.9]$} & [1.9] & {$[1.9 ; 1.9]$} \\
\hline LP dendritic filter $(\mathrm{Hz})$ & [1000] & [1000] & {$[50 ; 50]$} & [500] & {$[70 ; 70]$} \\
\hline \multicolumn{6}{|l|}{ Max Conductance (nS): } \\
\hline $\bar{g}_{N a}$ & [120] & [120] & [120] & [120] & [120] \\
\hline $\bar{g}_{K L T}$ & [5] & [10] & {$[20]$} & [25] & [5] \\
\hline $\bar{g}_{K H T}$ & [10] & [20] & [30] & [25] & [10] \\
\hline $\bar{g}_{H}$ & {$[0]$} & [5] & {$[0]$} & {$[0]$} & {$[0]$} \\
\hline$\overline{\boldsymbol{g}}_{L}$ & [2.9] & [2.9] & [2.9] & [2.9] & [2.9] \\
\hline \multicolumn{6}{|l|}{ Reversal Potentials (mV): } \\
\hline \begin{tabular}{|l|} 
\\
$N a$ \\
\end{tabular} & {$[+55]$} & {$[+55]$} & {$[+55]$} & {$[+55]$} & {$[+55]$} \\
\hline$E_{K}$ & {$[-80]$} & {$[-80]$} & {$[-80]$} & {$[-80]$} & {$[-80]$} \\
\hline \multirow{2}{*}{$\begin{array}{l}E_{H} \\
E_{L}\end{array}$} & $\mathrm{~N} / \mathrm{A}$ & {$[-43]$} & $\mathrm{N} / \mathrm{A}$ & $\mathrm{N} / \mathrm{A}$ & $\mathrm{N} / \mathrm{A}$ \\
\hline & {$[-62]$} & {$[-61]$} & {$[-59]$} & {$[-63]$} & {$[-64]$} \\
\hline \multicolumn{6}{|l|}{ Membrane Capacitance (pF): } \\
\hline$C_{m}$ & [1] & [1] & [1] & [1] & [1] \\
\hline Excitatory/lnhibitory output & N/A & Excitation & Excitation & Inhibition & MOC attenuation \\
\hline
\end{tabular}

LSR/HSR AN Fiber = Low/High Spontaneous Rate Auditory Nerve Fiber; LSR/HSR syn = Low/High Spontaneous Inner Hair Cell Synapse; VNTB = Ventral Nucleus of the Trapezoid Body. 'ipsi' and 'contra' is relative to the simulated ear or cell in question. $C F$ is the characteristic frequency of AN fiber tested and represents within-channel for input bandwidth. LP dendritic filter represents cut-off frequency of low-pass dendritic filter used to determine unitary synaptic conductance $\left(\bar{g}_{s y n}\right)$ at time,

$907 t$, as follows:

$$
\tau_{\text {Spiketocurrent }}=\frac{1}{\left(2 \pi f_{L P \text { dendritic filter }}\right)}
$$

$$
\bar{g}_{\text {syn }}(t)=\left(\frac{t-t_{0}}{\tau_{\text {spiketocurrent }}}\right) \exp \left(-\frac{t-t_{0}}{\tau_{\text {Spiketocurrent }}}\right)
$$

Where $t_{0}$ is the onset of the synaptic event. Current per input per spike was calculated by dividing Max synaptic current by Number of inputs. The above conductance and synaptic parameters are not absolute values in the physiological sense, instead mirroring key features of the physiology with a view to reproducing the time course of spiking activity and the qualitative differences of neural 
921 Table supplement 2. Subjects removed for CEOAEs inhibition analysis

922

\begin{tabular}{|l|l|l|l|l|}
\hline & Outliers & Below 6 dB snr & $\begin{array}{l}\text { Incomplete data } \\
\text { collection }\end{array}$ & Total $\mathrm{n}$ \\
\hline Active Natural & 4 & 20 & N/A & 25 \\
\hline Active Voc16 & 4 & 20 & N/A & 25 \\
\hline Active Voc12 & N/A & 20 & 27 & 25 \\
\hline Active Voc8 & N/A & 20 & N/A & 26 \\
\hline Passive Natural & N/A & 20 & N/A & 26 \\
\hline Passive Voc16 & N/A & 20 & 25 & 25 \\
\hline Passive Voc12 & N/A & 20 & N/A & 26 \\
\hline Passive Voc8 & N/A & 20 & N/A & 26 \\
\hline Active Natural & N/A & 19 & N/A & 28 \\
\hline Active BN10 & N/A & N/A & N/A & 29 \\
\hline Active BN5 & N/A & 6 & N/A & 28 \\
\hline Active SSN8 & N/A & 18 & N/A & 28 \\
\hline Active SSN3 & N/A & N/A & N/A & 29 \\
\hline Passive Natural & N/A & 6,16 & N/A & 27 \\
\hline Passive BN10 & N/A & N/A & N/A & 29 \\
\hline Passive BN5 & N/A & N/A & N/A & 29 \\
\hline Passive SSN8 & N/A & N/A & N/A & 29 \\
\hline Passive SSN3 & N/A & N/A & N/A & 29 \\
\hline & & & & \\
\hline
\end{tabular}

923

924

925

Table supplement 3. Subjects removed for ABRs analysis

\begin{tabular}{|l|l|l|l|l|}
\hline & Outliers & $\begin{array}{l}\text { Below noise } \\
\text { floor }\end{array}$ & $\begin{array}{l}\text { Incomplete data } \\
\text { collection }\end{array}$ & Total $\mathrm{n}$ \\
\hline Noise-vocoded-Wave V & 3 & 5 & 25,27 & 23 \\
\hline Noise-vocoded-Wave III & 3 & $5,12,16$ & 25,27 & 21 \\
\hline Noise-vocoded-Ratio & 3 & $5,12,16$ & 25,27 & 21 \\
\hline Babble Noise-Wave V & 10 & 16 & N/A & 27 \\
\hline Babble Noise-Wave III & 5,27 & 6,16 & N/A & 24 \\
\hline Babble Noise-Ratio & 5,19 & 6,16 & N/A & 25 \\
\hline Speech-Shaped noise-Wave V & 10 & 16 & N/A & 27 \\
\hline Speech-Shaped noise-Wave III & 5,27 & $6,13,16$ & N/A & 25 \\
\hline Speech-Shaped noise-Ratio & 5,19 & 6,16 & N/A & 25 \\
\hline
\end{tabular}


930 1. Lesica NA. Why do hearing aids fail to restore normal auditory perception? Trends Neurosci. $931 \quad 2018 ; 41: 174-185$.

932 2. Lehmann J, Christen N, Barilan YM, Gannot I. Age-related hearing loss, speech understanding 933 and cognitive technologies. Int J Speech Technol. 2021. doi:10.1007/s10772-021-09817-z

934 3. Chien W, Lin FR. Prevalence of hearing aid use among older adults in the United States. Arch 935 Intern Med. 2012;172: 292-293.

936 4. Simpson AN, Matthews LJ, Cassarly C, Dubno JR. Time From Hearing Aid Candidacy to

5. Irace AL, Sharma RK, Reed NS, Golub JS. Smartphone-Based Applications to Detect Hearing

6. Darrow KN, Maison SF, Liberman MC. Cochlear efferent feedback balances interaural sensitivity. Nat Neurosci. 2006;9: 1474-1476.

7. Grothe B, Pecka M. The natural history of sound localization in mammals--a story of neuronal

8. Smith DW, Keil A. The biological role of the medial olivocochlear efferents in hearing: inhibition. Front Neural Circuits. 2014;8: 116.

9. Ahveninen J, Jääskeläinen IP, Raij T, Bonmassar G, Devore S, Hämäläinen M, et al. Taskmodulated "what" and "where" pathways in human auditory cortex. Proc Natl Acad Sci U S A. 2006;103: 14608-14613.

10. Knudsen El. Fundamental components of attention. Annu Rev Neurosci. 2007;30: 57-78.

11. Mulders $\mathrm{WH}$, Robertson D. Evidence for direct cortical innervation of medial olivocochlear neurones in rats. Hear Res. 2000;144: 65-72.

12. Xiao Z, Suga N. Modulation of cochlear hair cells by the auditory cortex in the mustached bat. Nat Neurosci. 2002;5: 57-63.

13. Dragicevic CD, Aedo C, León A, Bowen M, Jara N, Terreros G, et al. The olivocochlear reflex strength and cochlear sensitivity are independently modulated by auditory cortex microstimulation. J Assoc Res Otolaryngol. 2015;16: 223-240.

14. Ashmore J, Avan P, Brownell WE, Dallos P, Dierkes K, Fettiplace R, et al. The remarkable cochlear amplifier. Hear Res. 2010;266: 1-17.

15. Almishaal A, Jennings SG. Effects of a precursor on amplitude modulation detection are consistent with efferent feedback. J Acoust Soc Am. 2016;139: 2155-2155.

16. Winslow RL, Sachs MB. Effect of electrical stimulation of the crossed olivocochlear bundle on auditory nerve response to tones in noise. J Neurophysiol. 1987;57: 1002-1021.

17. Guinan JJ Jr, Gifford ML. Effects of electrical stimulation of efferent olivocochlear neurons on cat auditory-nerve fibers. I. Rate-level functions. Hear Res. 1988;33: 97-113.

18. Faye-Lund $\mathrm{H}$. Projection from the inferior colliculus to the superior olivary complex in the albino rat. Anat Embryol . 1986;175: 35-52. 
19. Mulders WH, Robertson D. Effects on cochlear responses of activation of descending pathways from the inferior colliculus. Hear Res. 2000;149: 11-23.

20. Suthakar K, Ryugo DK. Descending projections from the inferior colliculus to medial olivocochlear efferents: Mice with normal hearing, early onset hearing loss, and congenital deafness. Hear Res. 2017;343: 34-49.

21. Guinan JJ Jr. Olivocochlear efferents: Their action, effects, measurement and uses, and the impact of the new conception of cochlear mechanical responses. Hear Res. 2018;362: 38-47.

22. de Boer J, Thornton ARD, Krumbholz K. What is the role of the medial olivocochlear system in speech-in-noise processing? J Neurophysiol. 2012;107: 1301-1312.

23. Mishra SK, Lutman ME. Top-down influences of the medial olivocochlear efferent system in speech perception in noise. PLoS One. 2014;9: e85756.

24. Mertes IB, Johnson KM, Dinger ZA. Olivocochlear efferent contributions to speech-in-noise recognition across signal-to-noise ratios. J Acoust Soc Am. 2019;145: 1529.

25. Kemp DT. Stimulated acoustic emissions from within the human auditory system. J Acoust Soc Am. 1978;64: 1386-1391.

26. Collet L, Kemp DT, Veuillet E, Duclaux R, Moulin A, Morgon A. Effect of contralateral auditory stimuli on active cochlear micro-mechanical properties in human subjects. Hear Res. 1990;43: 251-261.

27. Giraud AL, Garnier S, Micheyl C, Lina G, Chays A, Chéry-Croze S. Auditory efferents involved in speech-in-noise intelligibility. Neuroreport. 1997;8: 1779-1783.

28. Harkrider AW, Bowers CD. Evidence for a cortically mediated release from inhibition in the human cochlea. J Am Acad Audiol. 2009;20: 208-215.

29. Wagner W, Frey K, Heppelmann G, Plontke SK, Zenner H-P. Speech-in-noise intelligibility does not correlate with efferent olivocochlear reflex in humans with normal hearing. Acta Otolaryngol. 2008;128: 53-60.

30. Stuart A, Butler AK. Contralateral suppression of transient otoacoustic emissions and sentence recognition in noise in young adults. J Am Acad Audiol. 2012;23: 686-696.

31. Schaette R, McAlpine D. Tinnitus with a normal audiogram: physiological evidence for hidden hearing loss and computational model. J Neurosci. 2011;31: 13452-13457.

32. Kahneman D. Attention and effort. Prentice-Hall; 1973.

33. Meddis R. MAP-BS a Matlab Auditory Processing software platform for studying Auditory BrainStem activity. 2016 [cited 3 Jun 2020]. doi:10.13140/RG.2.2.30627.45603

34. Costalupes JA, Young ED, Gibson DJ. Effects of continuous noise backgrounds on rate response of auditory nerve fibers in cat. J Neurophysiol. 1984;51: 1326-1344.

35. Kujawa SG, Liberman MC. Synaptopathy in the noise-exposed and aging cochlea: Primary neural degeneration in acquired sensorineural hearing loss. Hear Res. 2015;330: 191-199.

36. Huet A, Desmadryl G, Justal T, Nouvian R, Puel J-L, Bourien J. The Interplay Between SpikeTime and Spike-Rate Modes in the Auditory Nerve Encodes Tone-In-Noise Threshold. J Neurosci. 2018;38: 5727-5738. 
1006

1007

1008

1009

1010

1011

1012

1013

1014

1015

1016

1017

1018

1019

1020

1021

1022

1023

1024

1025

1026

1027

1028

1029

1030

1031

1032

1033

1034

1035

1036

1037

1038

1039

1040

1041

1042

1043

1044

1045

1046

37. Heinz MG, Swaminathan J. Quantifying envelope and fine-structure coding in auditory nerve responses to chimaeric speech. J Assoc Res Otolaryngol. 2009;10: 407-423.

38. Liberman MC. Auditory-nerve response from cats raised in a low-noise chamber. J Acoust Soc Am. 1978;63: 442-455.

39. Winter IM, Palmer AR. Intensity coding in low-frequency auditory-nerve fibers of the guinea pig. J Acoust Soc Am. 1991;90: 1958-1967.

40. Heinz MG, Young ED. Response growth with sound level in auditory-nerve fibers after noiseinduced hearing loss. J Neurophysiol. 2004;91: 784-795.

41. Carney LH. Supra-Threshold Hearing and Fluctuation Profiles: Implications for Sensorineural and Hidden Hearing Loss. J Assoc Res Otolaryngol. 2018. doi:10.1007/s10162-018-0669-5

42. Encina-Llamas G, Harte JM, Dau T, Shinn-Cunningham B, Epp B. Investigating the effect of cochlear synaptopathy on envelope following responses using a model of the auditory nerve. J Assoc Res Otolaryngol. 2019;20: 363-382.

43. Pujol R. Cerveau auditif. [cited 10 Mar 2021]. Available: http://www.cochlea.eu/cerveau-auditif

44. Getzmann S, Falkenstein M, Wascher E. ERP correlates of auditory goal-directed behavior of younger and older adults in a dynamic speech perception task. Behav Brain Res. 2015;278: 435-445.

45. Grunwald T, Boutros NN, Pezer N, von Oertzen J, Fernández G, Schaller C, et al. Neuronal substrates of sensory gating within the human brain. Biol Psychiatry. 2003;53: 511-519.

46. Potts GF. An ERP index of task relevance evaluation of visual stimuli. Brain Cogn. 2004;56: 5-13.

47. Juottonen K, Revonsuo A, Lang H. Dissimilar age influences on two ERP waveforms (LPC and N400) reflecting semantic context effect. Brain Res Cogn Brain Res. 1996;4: 99-107.

48. Stuss DT, Picton TW, Cerri AM, Leech EE, Stethem LL. Perceptual closure and object identification: electrophysiological responses to incomplete pictures. Brain Cogn. 1992;19: 253-266.

49. Francis AL, MacPherson MK, Chandrasekaran B, Alvar AM. Autonomic Nervous System Responses During Perception of Masked Speech may Reflect Constructs other than Subjective Listening Effort. Front Psychol. 2016;7: 263.

50. Pichora-Fuller MK, Kramer SE, Eckert MA, Edwards B, Hornsby BWY, Humes LE, et al. Hearing Impairment and Cognitive Energy: The Framework for Understanding Effortful Listening (FUEL). Ear Hear. 2016;37 Suppl 1: 5S-27S.

51. Kutas M, Federmeier KD. Thirty years and counting: finding meaning in the N400 component of the event-related brain potential (ERP). Annu Rev Psychol. 2011;62: 621-647.

52. Puel JL, Bonfils P, Pujol R. Selective attention modifies the active micromechanical properties of the cochlea. Brain Res. 1988;447: 380-383.

53. Wittekindt A, Kaiser J, Abel C. Attentional modulation of the inner ear: a combined otoacoustic emission and EEG study. J Neurosci. 2014;34: 9995-10002.

54. Bowen M, Terreros G, Moreno-Gómez FN, Ipinza M, Vicencio S, Robles L, et al. The olivocochlear reflex strength in awake chinchillas is relevant for behavioural performance during visual selective attention with auditory distractors. Sci Rep. 2020;10: 14894. 
55. Kawase T, Delgutte B, Liberman MC. Antimasking effects of the olivocochlear reflex. II. Enhancement of auditory-nerve response to masked tones. J Neurophysiol. 1993;70: 25332549.

56. Marian V, Lam TQ, Hayakawa S, Dhar S. Spontaneous Otoacoustic Emissions Reveal an Efficient Auditory Efferent Network. J Speech Lang Hear Res. 2018;61: 2827-2832.

57. Saiz-Alía M, Miller P, Reichenbach T. Otoacoustic emissions evoked by the time-varying harmonic structure of speech. eNeuro. 2021. doi:10.1523/ENEURO.0428-20.2021

58. Lavie N. Distracted and confused?: selective attention under load. Trends Cogn Sci. 2005;9: 75-82.

59. Lilaonitkul W, Guinan JJ Jr. Human medial olivocochlear reflex: effects as functions of contralateral, ipsilateral, and bilateral elicitor bandwidths. J Assoc Res Otolaryngol. 2009;10: 459-470.

60. Boothalingam S, Purcell D, Scollie S. Influence of $100 \mathrm{~Hz}$ amplitude modulation on the human medial olivocochlear reflex. Neurosci Lett. 2014;580: 56-61.

61. Kalaiah MK, Nanchirakal JF, Kharmawphlang L, Noronah SC. Contralateral suppression of transient evoked otoacoustic emissions for various noise signals. Hearing, Balance and Communication. 2017;15: 84-90.

62. Lopez-Poveda EA, Eustaquio-Martín A, Stohl JS, Wolford RD, Schatzer R, Wilson BS. A Binaural Cochlear Implant Sound Coding Strategy Inspired by the Contralateral Medial Olivocochlear Reflex. Ear Hear. 2016;37: e138-48.

63. Terreros G, Delano PH. Corticofugal modulation of peripheral auditory responses. Front Syst Neurosci. 2015;9: 134.

64. Hausfeld L, Shiell M, Formisano E, Riecke L. Cortical processing of distracting speech in noisy auditory scenes depends on perceptual demand. Neuroimage. 2021;228: 117670.

65. Heggdal POL, Aarstad HJ, Brännström J, Vassbotn FS, Specht K. An fMRI-study on singlesided deafness: Spectral-temporal properties and side of stimulation modulates hemispheric dominance. Neuroimage Clin. 2019;24: 101969.

66. Khalfa S, Bougeard R, Morand N, Veuillet E, Isnard J, Guenot M, et al. Evidence of peripheral auditory activity modulation by the auditory cortex in humans. Neuroscience. 2001;104: 347358.

67. Maison SF, Liberman MC. Predicting vulnerability to acoustic injury with a noninvasive assay of olivocochlear reflex strength. J Neurosci. 2000;20: 4701-4707.

68. Taranda J, Maison SF, Ballestero JA, Katz E, Savino J, Vetter DE, et al. A point mutation in the hair cell nicotinic cholinergic receptor prolongs cochlear inhibition and enhances noise protection. PLoS Biol. 2009;7: e18.

69. Boero LE, Castagna VC, Terreros G, Moglie MJ, Silva S, Maass JC, et al. Preventing presbycusis in mice with enhanced medial olivocochlear feedback. Proc Natl Acad Sci U S A. 2020;117: 11811-11819.

70. Wiederhold ML, Kiang NY. Effects of electric stimulation of the crossed olivocochlear bundle on single auditory-nerve fibers in the cat. J Acoust Soc Am. 1970;48: 950-965.

71. Cooper NP, Guinan JJ Jr. Efferent-mediated control of basilar membrane motion. J Physiol. 2006;576: 49-54. 
72. Murugasu E, Russell IJ. The effect of efferent stimulation on basilar membrane displacement in the basal turn of the guinea pig cochlea. J Neurosci. 1996;16: 325-332.

73. Marrufo-Pérez MI, Eustaquio-Martín A, Lopez-Poveda EA. Adaptation to Noise in Human Speech Recognition Unrelated to the Medial Olivocochlear Reflex. J Neurosci. 2018;38: 41384145.

74. Brown GJ, Ferry RT, Meddis R. A computer model of auditory efferent suppression: implications for the recognition of speech in noise. J Acoust Soc Am. 2010;127: 943-954.

75. Clark NR, Brown GJ, Jürgens T, Meddis R. A frequency-selective feedback model of auditory efferent suppression and its implications for the recognition of speech in noise. J Acoust Soc Am. 2012;132: 1535-1541.

76. Yasin I, Drga V, Liu F, Demosthenous A, Meddis R. Optimizing Speech Recognition Using a Computational Model of Human Hearing: Effect of Noise Type and Efferent Time Constants. IEEE Access. 2020;8: 56711-56719.

77. Messing DP, Delhorne L, Bruckert E, Braida LD, Ghitza O. A non-linear efferent-inspired model of the auditory system; matching human confusions in stationary noise. Speech Commun. 2009;51: 668-683.

78. Mertes IB, Wilbanks EC, Leek MR. Olivocochlear Efferent Activity Is Associated With the Slope of the Psychometric Function of Speech Recognition in Noise. Ear Hear. 2018;39: 583-593.

79. Marrufo-Pérez MI, Sturla-Carreto DDP, Eustaquio-Martín A, Lopez-Poveda EA. Adaptation to Noise in Human Speech Recognition Depends on Noise-Level Statistics and Fast DynamicRange Compression. J Neurosci. 2020;40: 6613-6623.

80. Wojtczak M, Klang AM, Torunsky NT. Exploring the Role of Medial Olivocochlear Efferents on the Detection of Amplitude Modulation for Tones Presented in Noise. J Assoc Res Otolaryngol. 2019;20: 395-413.

81. Miller GA, Licklider JCR. The intelligibility of interrupted speech. J Acoust Soc Am. 1950;22: 167-173.

82. Cooke M. A glimpsing model of speech perception in noise. J Acoust Soc Am. 2006;119: $1562-1573$.

83. Li N, Loizou PC. Factors influencing glimpsing of speech in noise. J Acoust Soc Am. 2007;122: 1165-1172.

84. Jørgensen S, Ewert SD, Dau T. A multi-resolution envelope-power based model for speech intelligibility. J Acoust Soc Am. 2013;134: 436-446.

85. Relaño-lborra H, May T, Zaar J, Scheidiger C, Dau T. Predicting speech intelligibility based on a correlation metric in the envelope power spectrum domain. J Acoust Soc Am. 2016;140: 2670.

86. de Boer J, Thornton ARD. Neural correlates of perceptual learning in the auditory brainstem: efferent activity predicts and reflects improvement at a speech-in-noise discrimination task. $J$ Neurosci. 2008;28: 4929-4937.

87. Ding N, Simon JZ. Adaptive temporal encoding leads to a background-insensitive cortical representation of speech. J Neurosci. 2013;33: 5728-5735. 
88. Vanthornhout J, Decruy L, Wouters J, Simon JZ, Francart T. Speech Intelligibility Predicted from Neural Entrainment of the Speech Envelope. J Assoc Res Otolaryngol. 2018;19: 181191.

89. Brodbeck C, Jiao A, Hong LE, Simon JZ. Neural speech restoration at the cocktail party: Auditory cortex recovers masked speech of both attended and ignored speakers. PLoS Biol. 2020;18: e3000883.

90. Zeng F-G, Nie K, Liu S, Stickney G, Del Rio E, Kong Y-Y, et al. On the dichotomy in auditory perception between temporal envelope and fine structure cues. J Acoust Soc Am. 2004;116: $1351-1354$.

91. Lorenzi C, Gilbert G, Carn H, Garnier S, Moore BCJ. Speech perception problems of the hearing impaired reflect inability to use temporal fine structure. Proc Natl Acad Sci U S A. 2006;103: 18866-18869.

92. Shamma S, Lorenzi C. On the balance of envelope and temporal fine structure in the encoding of speech in the early auditory system. J Acoust Soc Am. 2013;133: 2818-2833.

93. Ding N, Chatterjee M, Simon JZ. Robust cortical entrainment to the speech envelope relies on the spectro-temporal fine structure. Neuroimage. 2014;88: 41-46.

94. Viswanathan V, Bharadwaj HM, Shinn-Cunningham B, Heinz MG. Evaluating human neural envelope coding as the basis of speech intelligibility in noise. J Acoust Soc Am. 2019;145: $1717-1717$.

95. Brown MC. Single-unit labeling of medial olivocochlear neurons: the cochlear frequency map for efferent axons. J Neurophysiol. 2014;111: 2177-2186.

96. Liberman LD, Liberman MC. Cochlear Efferent Innervation Is Sparse in Humans and Decreases with Age. J Neurosci. 2019;39: 9560-9569.

97. Forte $A E$, Etard $O$, Reichenbach $T$. The human auditory brainstem response to running speech reveals a subcortical mechanism for selective attention. Elife. 2017;6: e27203.

98. Brix R. The influence of attention on the auditory brain stem evoked responses. Preliminary report. Acta Otolaryngol. 1984;98: 89-92.

99. Lukas $\mathrm{JH}$. Human auditory attention: the olivocochlear bundle may function as a peripheral filter. Psychophysiology. 1980;17: 444-452.

100. Hillyard SA, Hink RF, Schwent VL, Picton TW. Electrical signs of selective attention in the human brain. Science. 1973;182: 177-180.

101. Fujiwara N, Nagamine T, Imai M, Tanaka T, Shibasaki H. Role of the primary auditory cortex in auditory selective attention studied by whole-head neuromagnetometer. Brain Res Cogn Brain Res. 1998;7: 99-109.

102. Mesgarani N, David SV, Fritz JB, Shamma SA. Mechanisms of noise robust representation of speech in primary auditory cortex. Proc Natl Acad Sci U S A. 2014;111: 6792-6797.

103. Rabinowitz NC, Willmore BDB, King AJ, Schnupp JWH. Constructing noise-invariant representations of sound in the auditory pathway. PLoS Biol. 2013;11: e1001710.

104. Kell AJE, McDermott JH. Invariance to background noise as a signature of non-primary auditory cortex. Nat Commun. 2019;10. doi:10.1038/s41467-019-11710-y 
105. Robinson BL, Harper NS, McAlpine D. Meta-adaptation in the auditory midbrain under cortical influence. Nat Commun. 2016;7: 13442.

1171 106. Shaheen LA, Slee SJ, David SV. Task Engagement Improves Neural Discriminability in the Auditory Midbrain of the Marmoset Monkey. J Neurosci. 2021;41: 284-297.

1173 107. Asokan MM, Williamson RS, Hancock KE, Polley DB. Sensory overamplification in layer 5 1174 auditory corticofugal projection neurons following cochlear nerve synaptic damage. Nat 1175 Commun. 2018;9: 2468.

108. Lehiste I, Peterson GE. Linguistic Considerations in the Study of Speech Intelligibility. J Acoust Soc Am. 1959;31: 280-286.

109. Rastle K, Harrington J, Coltheart M. 358,534 nonwords: the ARC Nonword Database. Q J Exp Psychol A. 2002;55: 1339-1362.

1180 110. Ritzer G, editor. Experimental Design. The Blackwell Encyclopedia of Sociology. Oxford, UK: John Wiley \& Sons, Ltd; 2007.

1182 111. Shannon RV, Zeng FG, Kamath V, Wygonski J, Ekelid M. Speech recognition with primarily 1183 temporal cues. Science. 1995;270: 303-304.

1184

1185

112. Keidser G, Ching T, Dillon H, Agung K, Brew C, Brewer S, et al. The National Acoustic Laboratories' (NAL) CDs of Speech and Noise for Hearing Aid Evaluation: Normative Data and Potential Applications. Australian and New Zealand Journal of Audiology. 2002;24: 16-35.

113. Boothalingam S, Purcell DW. Influence of the stimulus presentation rate on medial olivocochlear system assays. J Acoust Soc Am. 2015;137: 724-732.

114. Souza NN, Dhar S, Neely ST, Siegel JH. Comparison of nine methods to estimate earcanal stimulus levels. J Acoust Soc Am. 2014;136: 1768-1787.

115. Hood LJ, Berlin Cl, Hurley A, Cecola RP, Bell B. Contralateral suppression of transientevoked otoacoustic emissions in humans: intensity effects. Hear Res. 1996;101: 113-118.

116. Murata K, Ito S, Horikawa J, Minami S. The acoustic middle ear muscle reflex in albino rats. Hear Res. 1986;23: 169-183.

117. Liberman MC, Guinan JJ Jr. Feedback control of the auditory periphery: anti-masking effects of middle ear muscles vs. olivocochlear efferents. J Commun Disord. 1998;31: 47182; quiz 483; 553.

118. Lee DJ, de Venecia RK, Guinan JJ Jr, Brown MC. Central auditory pathways mediating the rat middle ear muscle reflexes. Anat Rec A Discov Mol Cell Evol Biol. 2006;288: 358-369.

119. Oostenveld R, Fries P, Maris E, Schoffelen J-M. FieldTrip: Open source software for advanced analysis of MEG, EEG, and invasive electrophysiological data. Comput Intell Neurosci. 2011;2011: 156869.

120. Woodman GF. A brief introduction to the use of event-related potentials in studies of perception and attention. Atten Percept Psychophys. 2010;72: 2031-2046.

121. Don M, Elberling C. Evaluating residual background noise in human auditory brain-stem responses. J Acoust Soc Am. 1994;96: 2746-2757.

122. Faul F, Erdfelder E, Lang A-G, Buchner A. G*Power 3: a flexible statistical power analysis 1209 program for the social, behavioral, and biomedical sciences. Behav Res Methods. 2007;39: 175-191. 
1210 123. Liberman MC. Response properties of cochlear efferent neurons: monaural vs. binaural stimulation and the effects of noise. J Neurophysiol. 1988;60: 1779-1798.

1212 124. Joris PX, Louage DH, Cardoen L, van der Heijden M. Correlation index: a new metric to quantify temporal coding. Hear Res. 2006;216-217: 19-30.

1214 125. Rallapalli VH, Heinz MG. Neural Spike-Train Analyses of the Speech-Based Envelope Power Spectrum Model: Application to Predicting Individual Differences with Sensorineural Hearing Loss. Trends in Hearing. 2016;20: 2331216516667319.

1217 126. Aubanel, V., Davis, C. Kim, J. The MAVA corpus. 2017. doi:10.4227/139/59a4c21a896a3 